IUCrJ

ISSN 2052-2525

PHYSICS|FELS

Received 27 February 2017

Accepted 13 April 2017

Edited by T. Ishikawa, Harima Institute, Japan

‡ These authors contributed equally.

Keywords: serial millisecond crystallography; synchrotron radiation; Advanced Photon

Source; high-viscosity injector.

PDB references: adenosine receptor $A_{2 A} A R$, 5uvi; lysozyme, 5uvj; phycocyanin, 5uvk; proteinase K, 5 uvl

Supporting information: this article has supporting information at www.iucrj.org

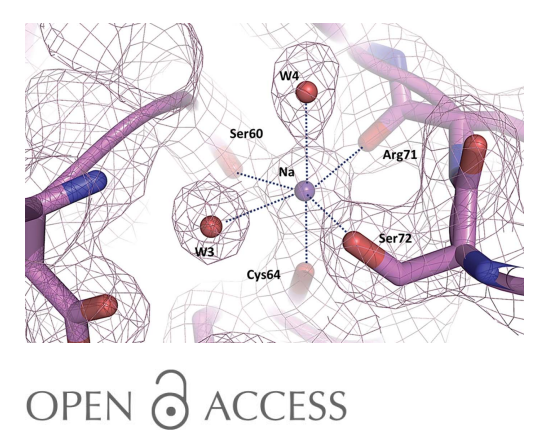

\section{Serial millisecond crystallography of membrane and soluble protein microcrystals using synchrotron radiation}

Jose M. Martin-Garcia, ${ }^{a} \ddagger$ Chelsie E. Conrad, ${ }^{a, b} \neq$ Garrett Nelson, ${ }^{a, c} \neq$ Natasha Stander, , ,c Nadia A. Zatsepin,, ${ }^{\text {a,c }}$ James Zook, ${ }^{a}$ Lan Zhu, James Geiger, ${ }^{a}$ Eugene Chun, ${ }^{a}$ David Kissick, ${ }^{d}$ Mark C. Hilgart, ${ }^{d}$ Craig Ogata, ${ }^{d}$ Andrii Ishchenko, ${ }^{\text {e Nirupa }}$ Nagaratnam, ${ }^{a}$ Shatabdi Roy-Chowdhury, ${ }^{a}$ Jesse Coe, ${ }^{a}$ Ganesh Subramanian, a,c Alexander Schaffer, ${ }^{a}$ Daniel James, ${ }^{f}$ Gihan Ketwala, ${ }^{c}$ Nagarajan Venugopalan, ${ }^{d}$ Shenglan Xu, ${ }^{d}$ Stephen Corcoran, ${ }^{d}$ Dale Ferguson, ${ }^{d}$ Uwe Weierstall, ${ }^{a, c}$ John C. H. Spence, ${ }^{c}$ Vadim Cherezov, ${ }^{\text {e Petra Fromme, }}{ }^{\mathrm{a} *}$ Robert F. Fischetti ${ }^{\mathrm{d} *}$ and Wei Liu ${ }^{\mathrm{a} *}$

\footnotetext{
aschool of Molecular Sciences and Biodesign Center for Applied Structural Discovery, Biodesign Institute, Arizona State University, Tempe, AZ 85287, USA, 'b Structural Biophysics Laboratory, National Cancer Institute, Frederick, MD 21702, USA, ' Department of Physics, Arizona State University, PO Box 871504, Tempe, AZ 85287, USA, 'dAdvanced Photon Source, Argonne National Laboratory, 9700 South Cass Avenue, Lemont, IL 60439, USA, 'Department of Chemistry, Bridge Institute, University of Southern California, 3430 South Vermont Avenue, MC 3303, Los Angeles, CA 90089, USA, and ${ }^{\mathbf{f}}$ Paul Scherrer Institute, 5232 Villigen, Switzerland. *Correspondence e-mail: pfromme@asu.edu, rfischetti@anl.gov, w.liu@asu.edu
}

Crystal structure determination of biological macromolecules using the novel technique of serial femtosecond crystallography (SFX) is severely limited by the scarcity of X-ray free-electron laser (XFEL) sources. However, recent and future upgrades render microfocus beamlines at synchrotron-radiation sources suitable for room-temperature serial crystallography data collection also. Owing to the longer exposure times that are needed at synchrotrons, serial data collection is termed serial millisecond crystallography (SMX). As a result, the number of SMX experiments is growing rapidly, with a dozen experiments reported so far. Here, the first high-viscosity injector-based SMX experiments carried out at a US synchrotron source, the Advanced Photon Source (APS), are reported. Microcrystals $(5-20 \mu \mathrm{m})$ of a wide variety of proteins, including lysozyme, thaumatin, phycocyanin, the human $\mathrm{A}_{2 \mathrm{~A}}$ adenosine receptor $\left(\mathrm{A}_{2 \mathrm{~A}} \mathrm{AR}\right)$, the soluble fragment of the membrane lipoprotein Flpp3 and proteinase $\mathrm{K}$, were screened. Crystals suspended in lipidic cubic phase (LCP) or a high-molecular-weight poly(ethylene oxide) (PEO; molecular weight $8000000)$ were delivered to the beam using a high-viscosity injector. In-house data-reduction (hit-finding) software developed at APS as well as the SFX datareduction and analysis software suites Cheetah and CrystFEL enabled efficient on-site SMX data monitoring, reduction and processing. Complete data sets were collected for $\mathrm{A}_{2 \mathrm{~A}} \mathrm{AR}$, phycocyanin, Flpp3, proteinase $\mathrm{K}$ and lysozyme, and the structures of $\mathrm{A}_{2 \mathrm{~A}} \mathrm{AR}$, phycocyanin, proteinase $\mathrm{K}$ and lysozyme were determined at $3.2,3.1,2.65$ and $2.05 \AA$ resolution, respectively. The data demonstrate the feasibility of serial millisecond crystallography from 5-20 $\mu \mathrm{m}$ crystals using a high-viscosity injector at APS. The resolution of the crystal structures obtained in this study was dictated by the current flux density and crystal size, but upcoming developments in beamline optics and the planned APS-U upgrade will increase the intensity by two orders of magnitude. These developments will enable structure determination from smaller and/or weakly diffracting microcrystals.

\section{Introduction}

Despite the deposition of over 115000 crystal structures of biological macromolecules in the Protein Data Bank (PDB; http://www.rcsb.org), traditional techniques of macromolecular X-ray crystallography have always suffered from 
two major bottlenecks: the production of large, well diffracting crystals and radiation damage. The former has been especially prevalent in the structure determination of membrane proteins and large complexes, which can require months or even years of devoted effort to optimize conditions for the growth of crystals of sufficient quality, while the latter has hindered progress in the structural determination of radiationsensitive proteins. For decades, the effect of radiation damage in macromolecular crystallography has been addressed by cryocooling crystals (Low et al., 1966; Macchi, 2012; Pflugrath, 2004), thus extending their lifetime during X-ray beam exposure. However, third-generation synchrotrons produce such intense microfocus X-ray beams that rapid photodamage can rapidly accumulate even under cryogenic conditions (Ravelli \& Garman, 2006). Both the crystal-size and radiation-damage limitations have now been overcome by $\mathrm{X}$-ray free-electron lasers (XFELs), the brightest X-ray sources, which are capable of producing extremely intense femtosecond X-ray pulses. Taking advantage of these unique properties, a new method of crystallography has emerged, termed serial femtosecond crystallography (SFX; Chapman et al., 2011). In SFX, hundreds of thousands of nanocrystals and microcrystals suspended in their mother liquor or in a viscous carrier are delivered to the X-ray beam in a jet. The duration of exposure per crystal is so short (typically $40 \mathrm{fs}$ ) that diffraction can be recorded by a fast, integrating detector before the beam destroys the crystal, thereby outrunning radiation damage (Chapman et al., 2011; Neutze et al., 2000). The high intensity of the XFEL pulses used in SFX eliminates the need to grow large crystals. Inspired by this novel methodology, a number of innovations such as nanocrystallization and microcrystallization techniques (Kupitz et al., 2014; Liu et al., 2014; Redecke et al., 2013), serial sample-delivery methods (Ayvazyan et al., 2006; Feld et al., 2015; Huang et al., 2015; Sierra et al., 2012; Weierstall et al., 2012, 2014), fastreadout detector technology (e.g. CSPAD) and rapid, highthroughput data-reduction and data-processing software have been developed (Barty et al., 2014; Kirian et al., 2010, 2011; Sauter, 2015; Sawaya et al., 2014; White et al., 2012, 2013, 2016). Consequently, numerous SFX crystal structures of membrane and soluble proteins have now been solved and deposited in the PDB (for a review, see Martin-Garcia et al., 2016), showing the increasing utility of this technology to structural biologists.

Despite the advantages mentioned above, the use of SFX is severely limited by the scarcity of XFEL facilities. Currently, only two XFELs are operational in the world: the Linac Coherent Light Source (LCLS) at the SLAC National Accelerator Laboratory, Stanford, California, USA and the SPring-8 Angstrom Compact Free-Electron Laser (SACLA) in Japan. Unlike synchrotrons, the circular design of which allows the placement of many beamlines around the storage ring, XFELs are limited to only a few beamlines, only one of which can collect diffraction data at any given time, owing to their linear design. Even though several XFEL sources are currently under construction (PAL in South Korea, SwissFEL in Switzerland and the European XFEL in Germany) and are expected to enter the commissioning phase in 2017, the total number of XFELs will still be too low to meet the growing needs of the structural biology community compared with the numerous synchrotron facilities currently available worldwide (over 30 synchrotrons; http://www.esrf.eu/UsersAndScience/ Links/Synchrotrons). To overcome this limitation, the structural biology community has begun to adopt the serial crystallography approach at the microfocus beamlines of third-generation synchrotron-radiation sources, which produce bright micrometre-sized X-ray beams and are equipped with fast-readout detectors (e.g. PILATUS and EIGER). However, since the brilliance of synchrotrons is not as high as that of XFELs, at least a millisecond exposure is required to produce sufficiently strong diffraction. Here, the microcrystals are delivered either loaded into fixed-target devices or continuously in single file across the beam in a viscous host medium. Because a goniometer is not used, a sufficiently viscous medium is important to prevent significant rotation of the crystals during exposure. The change in diffraction conditions is therefore negligible if the rotational diffusion time of the crystal in the medium is much longer than the exposure time. Thus, this method has been named serial millisecond crystallography (SMX). Over the last three years, several SMX trials have been published (Botha et al., 2015; Coquelle et al., 2015; Gati et al., 2014; Hasegawa et al., 2016; Heymann et al., 2014; Huang et al., 2015, 2016; Murray et al., 2015; Nogly et al., 2015; Stellato et al., 2014; Zander et al., 2015).

One of the major concerns in adopting the serial approach at synchrotrons was radiation damage. It has been reported that $0.3 \mathrm{MGy}$ is the radiation-dose limit beyond which the information collected from a single crystal at room temperature is compromised (Nave \& Garman, 2005). At XFELs, the pulse duration (femtoseconds) is sufficiently short such that the effects of radiation damage can be mitigated. An XFEL femtosecond pulse leads to photo-ionization of the inner core shell electrons, producing 'hollow atoms', in less than $10 \mathrm{fs}$ (Hau-Riege \& Bennion, 2015). Molecules are then destroyed by Coulomb explosion, which terminates diffraction before secondary radiation damage can take place. For this reason, even though XFELs produce extremely brilliant X-rays, SFX data show sufficiently reduced radiation damage to allow the collection of data at doses in excess of $3 \mathrm{GGy}$ (Lomb et al., 2011), which is over 10000 times the estimated tolerable dose at room temperature (Nave \& Garman, 2005). At synchrotron sources, the low monochromatic photon flux along with the quasi-continuous beam-operation mode lead to an exposure timescale per crystal/image in the millisecond range, which is not fast enough to outrun primary damage. Here, secondary radiation damage plays a major role as free radicals reduce molecules, leading to the breakage of chemical bonds, which finally destroys the molecules and weakens crystal contacts on much longer timescales than the Coulomb explosion observed with XFEL femtosecond pulses. However, a recent study has demonstrated that for the same total dose, secondary radiation damage owing to diffusion of free radicals can be reduced by increasing the intensity and inversely decreasing the 
exposure time (Owen et al., 2012). Another factor that has contributed to the success of SMX at synchrotrons is the recent improvement in sample-delivery methods (for example the high-viscosity injector), and the high efficiency achieved with fast-readout detectors that can collect data in a shutterless mode. High-viscosity injectors require reduced sample volumes compared with liquid injectors (for example the gas dynamic virtual nozzle; GDVN; Weierstall et al., 2014). In addition, fast-flowing liquid injectors are impractical for SMX experiments because the crystal transit time across the beam would be in the submillisecond range and thus the crystal would not be exposed to sufficient flux to diffract strongly. A large variety of sample-delivery methods have been successfully applied, including delivering crystals by flowing them through capillaries (Stellato et al., 2014), high-viscosity injectors (Botha et al., 2015; Nogly et al., 2015) and novel fixedtarget systems (Coquelle et al., 2015; Gati et al., 2014; Hasegawa et al., 2016; Huang et al., 2015; Murray et al., 2015).

With the intention of making the serial crystallography approach more broadly accessible to the structural biology community, we implemented SMX at the GM/CA 23-ID-D beamline at the Advanced Photon Source (APS), a thirdgeneration synchrotron source at Argonne National Laboratory, Chicago, Illinois, USA. A set of proteins was chosen including membrane proteins, soluble proteins and a multiprotein cofactor complex. The membrane protein was the human $\mathrm{A}_{2 \mathrm{~A}}$ adenosine receptor containing the BRIL fusion protein in the third intracellular loop $\left(\mathrm{A}_{2 \mathrm{~A}} \mathrm{AR}\right) . \mathrm{A}_{2 \mathrm{~A}} \mathrm{AR}$ is one of the best-studied human G-protein coupled receptors (GPCRs) for which a structure has been determined previously (Batyuk et al., 2016; Liu et al., 2012). The set of soluble proteins that we tested include Francisella tularensis SCHUS4 Flpp3 soluble domain, proteinase $\mathrm{K}$, thaumatin and lysozyme, and the multiprotein cofactor antenna complex phycocyanin (PC). PC is a cyanobacterial antenna protein that forms part of the phycobilisome light-harvesting complex, which channels excitation energy to photosystem II. The PC complex forms a disk-like trimer in which each monomer is composed of two subunits, $\alpha$ and $\beta$ (Schirmer et al., 1985), where each subunit binds three chromophores. Flpp3 is the soluble domain of a membrane lipoprotein located in the outer membrane of $F$. tularensis, which is the causative agent of the disease tularemia. Flpp3 has been identified as a virulence determinant (Su et al., 2007), and its atomic resolution structure has recently been determined by NMR (Zook et al., 2015); however, a crystallographic structure has not yet been reported. Proteinase $\mathrm{K}$ is a commercially available enzyme belonging to the family of serine proteases, which has been used in numerous studies to identify disordered regions in intrinsically disordered proteins (IDPs; Csizmók et al., 2005; Denning et al., 2003; Marín et al., 2012; Martin-Garcia et al., 2014; Nyarko et al., 2004). Thaumatin and lysozyme were also included as they crystallize rapidly and easily, and are therefore frequently used as model systems in protein crystallization studies and many proof-of-principle studies.

The goal of this study was not to determine the structures of these proteins, but to evaluate the feasibility of SMX experiments by using the current capabilities of the GM/CA 23-ID-D beamline in combination with various delivery methods. For this, microrystals in a size range from 5 to $20 \mu \mathrm{m}$ were grown and delivered to the X-ray beam using a highviscosity injector (Weierstall et al., 2014). We also tested two viscous media as crystal carriers: lipidic cubic phase (LCP) and poly(ethylene oxide) (PEO), a gel polymer with a high molecular weight (8000 000). Additionally, we developed a new program at APS for real-time data monitoring and reduction, as well as a new version of the Cheetah software (Cheetah-cbf) for data reduction (hit-finding) that reads raw images from the PILATUS detector. In this proof-of-principle study, we determined the feasibility of structure determination of several proteins, including $\mathrm{A}_{2 \mathrm{~A}} \mathrm{AR}, \mathrm{PC}$, Flpp3, proteinase $\mathrm{K}$ and lysozyme, using SMX. Much faster data collection will be possible using the upgraded high-brightness synchrotron sources and faster detectors that are under way or planned at many facilities.

\section{Materials and methods}

\subsection{Microcrystal sample preparation}

A total of six proteins were screened in this study: the human $\mathrm{A}_{2 \mathrm{~A}}$ adenosine receptor containing the BRIL fusion protein in the third intracellular loop $\left(\mathrm{A}_{2 \mathrm{~A}} \mathrm{AR}\right)$; $\mathrm{PC}$ from Thermosynechococcus elongatus; Flpp3 from F. tularensis; chicken egg-white lysozyme; thaumatin from Thaumatococcus daniellii; and proteinase $\mathrm{K}$ from Tritirachium album.

Protein production and crystal sample preparations of all proteins screened were as follows. Expression, purification and crystallization of human $\mathrm{A}_{2 \mathrm{~A}} \mathrm{AR}$ were performed as described previously (Batyuk et al., 2016; Liu et al., 2012). Briefly, concentrated protein $\left(25 \mathrm{mg} \mathrm{ml}^{-1}\right)$ was reconstituted in LCP by mixing with molten monoolein/cholesterol [9:1(w/w)] using a syringe mixer (Cheng et al., 1998). Crystals of approximately $5 \mu \mathrm{m}$ were obtained in Hamilton syringes by injecting $6 \mu \mathrm{l}$ of protein-LCP mixture into a $100 \mu$ l syringe filled with $60 \mu \mathrm{l}$ of a precipitant solution consisting of $0.1 \mathrm{M}$ sodium citrate $\mathrm{pH} 5.0,32 \%$ PEG 400, $0.075 M$ sodium thiocyanate. Flpp3 was purified as described previously (Zook et al., 2015). 10-15 $\mu \mathrm{m}$ crystals of Flpp3 were grown by incubating the protein at $20 \mathrm{mg} \mathrm{ml}^{-1}$ in a low ionic strength buffer $\left(0.05 M \mathrm{NaCl}, 0.02 M\right.$ Tris $\mathrm{pH}$ 6.4) containing $1 \mathrm{U} \mathrm{ml}^{-1}$ factor Xa protease to cleave the His tag. Chicken egg-white lysozyme (catalog no. 62970, Sigma) crystals of sizes between 5 and $10 \mu \mathrm{m}$ were grown using the batch and vapor-diffusion methods. For the batch method, $40 \mu \mathrm{l}$ of lysozyme at $50 \mathrm{mg} \mathrm{ml}^{-1}$ in $0.02 \mathrm{M}$ sodium acetate $\mathrm{pH} 4.6$ was mixed with $200 \mu \mathrm{l}$ of a precipitant solution consisting of $18 \%(w / v) \mathrm{NaCl}$, $6 \%(w / v)$ PEG 6000, $0.1 M$ sodium acetate $\mathrm{pH}$ 3.0. Lysozyme microcrystals formed immediately at room temperature and were centrifuged at $500 \mathrm{~g}$ for $5 \mathrm{~min}$. For the vapor-diffusion method, lysozyme was crystallized from a kit purchased from Hampton Research (catalog no. HR7-108). A protein stock solution was made at a concentration of $100 \mathrm{mg} \mathrm{ml}^{-1}$ in deionized water. Hanging drops consisted of equal volumes of 
either 80 or $100 \mathrm{mg} \mathrm{ml}^{-1}$ protein solution and reservoir solution consisting of $30 \%(w / v)$ PEG 5000 monomethyl ether, 1.0 $M$ sodium chloride, $0.05 M$ sodium acetate trihydrate $\mathrm{pH}$ 4.6. Thaumatin was purchased from Sigma (catalog No. T7638) to grow microcrystals of $\sim 20 \mu \mathrm{m}$ in size using the vapordiffusion method at room temperature by mixing equal volumes of a protein solution at $40 \mathrm{mg} \mathrm{ml}^{-1}$ and a precipitant solution consisting of $0.1 \mathrm{M}$ sodium citrate $\mathrm{pH}$ 5.6, $0.75 \mathrm{M}$ potassium/sodium tartrate. Proteinase K (catalog No. P2308, Sigma) was crystallized using the batch method by mixing equal volumes of a protein solution at $40 \mathrm{mg} \mathrm{ml}^{-1}$ in $0.02 \mathrm{M}$ MES pH 6.5 and a precipitant solution composed of $0.1 \mathrm{M}$ MES pH 6.5, 0.5 $M$ sodium nitrate, $0.1 M$ calcium chloride. Crystals between 10 and $15 \mu \mathrm{m}$ in size appeared after $20 \mathrm{~min}$ incubation at $20^{\circ} \mathrm{C}$. PC was isolated from T. elongatus cells and purified as described previously (Fromme et al., 2015). PC crystals $(\sim 20 \mu \mathrm{m})$ were produced at $4^{\circ} \mathrm{C}$ using the batch method at a starting protein concentration of $20 \mathrm{mg} \mathrm{ml}^{-1}$ and using 0.075 $M$ HEPES pH 7.0, $0.02 M \mathrm{MgCl}_{2}, 9 \%(w / v)$ PEG 3350 as precipitant. Crystals were grown at $4{ }^{\circ} \mathrm{C}$, appeared

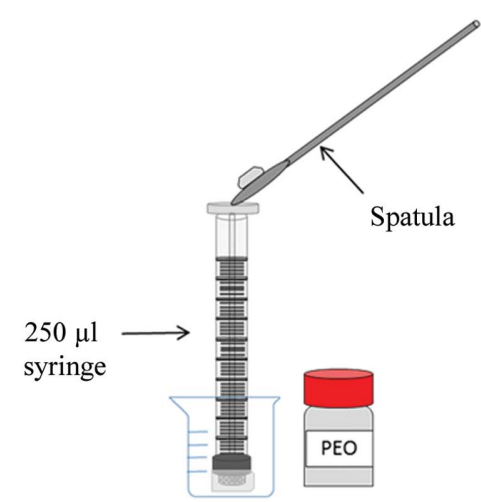

(a)
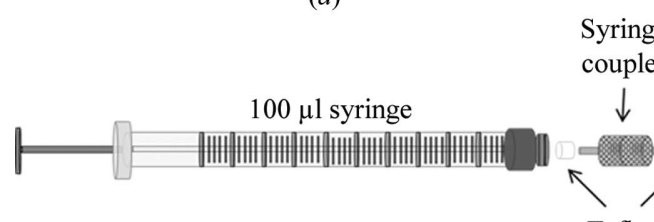

Teflon

ferrules

(c)

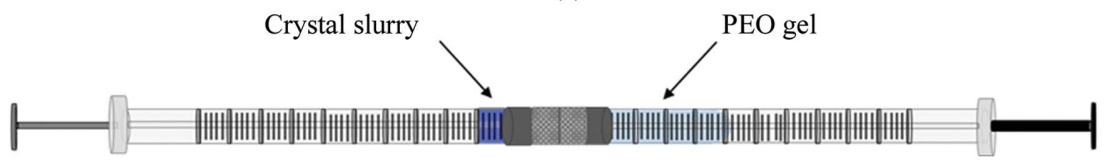

(d)

Figure 1

Diagram depicting the procedure used to prepare the poly(ethylene oxide) (PEO) gel and embed the crystals within it. (a) $0.6 \mathrm{mg}$ of PEO is weighed into the back end of a $250 \mu \mathrm{l}$ syringe and the PEO powder is then evenly distributed throughout the syringe by tapping the syringe while the syringe is horizontal (not shown). (b) The crystal precipitant $(50 \mu \mathrm{l})$ is then added via a pipette to the back end of the syringe and the plunger is inserted. (c) The $250 \mu \mathrm{l}$ syringe containing the precipitant solution and PEO is then connected to a $100 \mu \mathrm{l}$ syringe via a syringe coupler (Cheng et al., 1998) and the PEO is mixed until the suspension becomes clear by pushing the plungers of each syringe back and forth. The desired amount of PEO gel is then pushed into the $100 \mu \mathrm{l}$ syringe and the syringe coupler is disconnected. $(d)$ A second $100 \mu l$ syringe containing a concentrated slurry of crystals is connected to the $100 \mu \mathrm{l}$ syringe containing the PEO gel and the crystals are then embedded into the gel by pushing the plungers back and forth until the mixture is homogenous. within $12 \mathrm{~h}$ and were pooled together before mixing with the viscous media. Except for $\mathrm{A}_{2 \mathrm{~A}} \mathrm{AR}$, all crystallization setups were carried out onsite at the experimental laboratory of GM/CA beamline 23-ID-D.

\subsection{Mixing crystals with the injection carrier media}

$\mathrm{A}_{2 \mathrm{~A}} \mathrm{AR}$ crystals were grown in LCP and injected directly into the X-ray beam (LCP-embedded microcrystals of $\mathrm{A}_{2 \mathrm{~A}} \mathrm{AR}$ are shown in Supplementary Fig. S1). For the other proteins, two viscous media were used as carriers to deliver the crystals into the X-ray beam path. LCP was used to embed previously grown crystals of lysozyme (Supplementary Fig. S1), thaumatin and Flpp3. Crystal suspensions of approximately $20 \mu \mathrm{l}$ were pooled together and centrifuged at $500 \mathrm{~g}$ for $5 \mathrm{~min}$. The supernatant was removed and the remaining microcrystal suspension was mixed with molten monoolein lipid in a pide of $3: 2(v: v)$ using a dual-syringe lipid mixer until a homogeneous and transparent LCP was formed (Caffrey \& Cherezov, 2009; Cheng et al., 1998). PEO was used for the first time as a carrier to suspend previously formed crystals of Flpp3, PC and proteinase K. Fig. 1 shows the setup with which the crystals were embedded into the PEO medium. Prior to mixing with PEO, all crystal suspensions were centrifuged at $500 \mathrm{~g}$ for $5 \mathrm{~min}$ at $20^{\circ} \mathrm{C}$. A $13 \%(w / v)$ PEO gel was prepared by inserting $6.5 \mathrm{mg}$ PEO powder (Sigma-Aldrich) into the back end of a $250 \mu \mathrm{l}$ syringe with a ferrule in place and with Parafilm covering the removable needle end. Subsequently, $50 \mu \mathrm{l}$ of the corresponding precipitant solution was pipetted into the back end of the syringe, the plunger was inserted and the syringe was flipped upside down and moved up and down to remove air and to dissolve the PEO. To achieve a homogeneous PEO suspension, a $100 \mu \mathrm{l}$ syringe was connected to the $250 \mu \mathrm{l}$ syringe using a syringe coupler (Cheng et al., 1998) and the plungers of both syringes were then pushed back and forth until a fully clear suspension was achieved. In another $100 \mu \mathrm{l}$ syringe, the desired volume of crystals was drawn from the crystal pellet. Finally, a 4:1 volumetric ratio of $\mathrm{PEO}$ gel and concentrated crystals were mixed by pushing the crystals and gel 
through a syringe coupler (Cheng et al., 1998) back and forth until homogeneity was achieved. Supplementary Fig. S2 shows $\mathrm{PC}$ and proteinase $\mathrm{K}$ crystals after being mixed with the PEO gel.

In all cases the crystal concentration was adjusted so that mainly single-crystal hits were observed. The crystal mixtures were loaded directly from the Hamilton syringe into the LCP injector sample reservoir.

\subsection{Serial data collection on the GM/CA 23-ID-D beamline}

Serial data collection was performed on the GM/CA 23-ID-D beamline at the APS. The GM/CA beamline is equipped with a quad-minibeam collimator that provides high-intensity beams between 5 and $20 \mu \mathrm{m}$ in diameter with a scatter-guard aperture (Fischetti et al., 2009; Sanishvili et al., 2008); the JBluIce-EPICS GUI software allows users to fully exploit the rapid beam-size change and various models of data collection (Hilgart et al., 2011; Pothineni et al., 2014; Stepanov

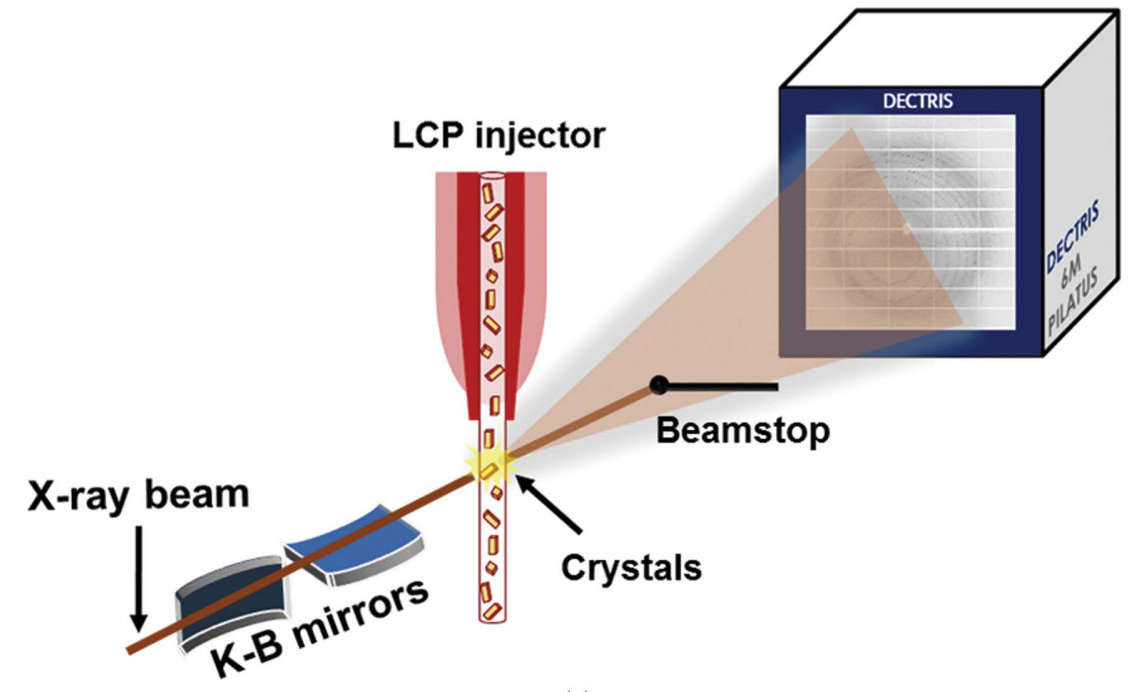

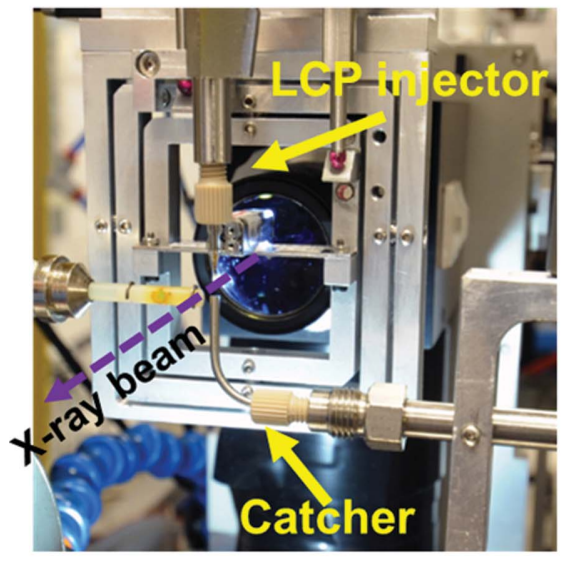

(b) (a)

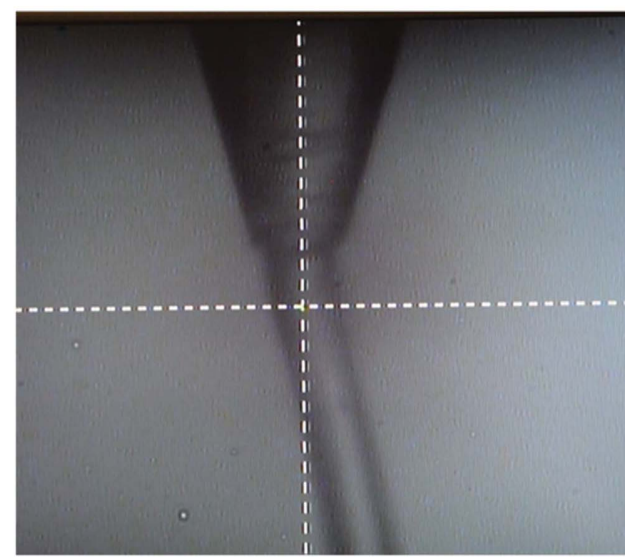

(c)
Figure 2

Experimental setup at the GM/CA 23-ID-D beamline. (a) Schematic diagram of the setup. (b) LCP injector (Weierstall et al., 2014) mounted on translation stages (not shown). The catcher is also shown. (c) View of the LCP stream extruding out of a $50 \mu \mathrm{m}$ glass capillary nozzle. The intersection point of the two white dashed lines indicates the position of the X-ray beam. et al., 2011). A new optical layout has been designed and will be installed shortly to produce intense microfocused beams as small as $1 \mu \mathrm{m}$ (Fischetti et al., 2013).

Microcrystal/carrier suspensions were injected to the intersection with the X-ray beam using the viscous LCP injector (Weierstall et al., 2014) with a $50 \mu \mathrm{m}$ inner diameter fused silica capillary ('nozzle'). This type of nozzle was chosen according to the crystal size to avoid shearing and breakage of the crystals during injection and to avoid nozzle clogging, as well as to minimize the background scattering from crystal carrier streams. For all our experiments a $20 \mu \mathrm{l}$ reservoir was used. The injector was mounted on translation stages to align the jet perpendicular to the X-ray beam path (Fig. 2). The crystal carrier stream was extruded out of the nozzle by a pressure that varied between 0.2 and $1 \mathrm{MPa}$ depending on the flow rate of the sample, which was also varied depending on the sample composition and the observed diffraction. A nitrogen-gas sheath was introduced at the point of extrusion to prevent curling of the viscous medium stream. An in-line highresolution microscope was used to align the nozzle to the beam and to observe the stream. A sample catcher consisting of a small diameter hyperdermic tube was placed opposite to the jet to catch the extruded waste sample. The catcher was connected to a vacuum pump via Tygon tubing with an inline particulate trap to protect the pump. The small tube tended to clog and was replaced by a $\frac{11}{4}$ tube. A schematic of the experimental setup used at the GM/CA 23-ID-D beamline is shown in Fig. 2.

During our two experiments, the beam generated by the undulator was monochromated to $0.02 \%$ bandwidth using a $\mathrm{Si}(111)$ monochromator and focused using Kirkpatrick-Baez (K-B) mirrors. The beamline operated at an energy of $12 \mathrm{keV}$ (wavelength $1.03 \AA$ ) and the flux at the focus using a $10 \mu \mathrm{m}$ (FWHM) beam size was 3.0-4.1 $\times$ $10^{11}$ photons s$^{-1}$. Tens to hundreds of thousands of single-shot diffraction patterns of randomly oriented microcrystals were recorded on a PILATUS3 $6 \mathrm{M}$ detector running in a continuous shutterless mode at a readout rate of $10 \mathrm{~Hz}$ (100 ms exposure time) while crystals were passing through the beam. The sample-to-detector distance varied between 300 and $550 \mathrm{~mm}$ depending on the sample diffraction quality. Although the crystal flow rate was varied based on the observed diffraction, most measurements were performed at an average flow rate which varied from 17 to $182 \mathrm{nl} \mathrm{min}^{-1}$ depending on the 
Table 1

SMX data-collection statistics.

Values in parentheses are for the highest resolution shell.

\begin{tabular}{|c|c|c|c|c|c|}
\hline & $\mathrm{A}_{2 \mathrm{~A}} \mathrm{AR}$ & Lysozyme & Phycocyanin & Flpp3 & Proteinase K \\
\hline Viscous medium & LCP & LCP & $\mathrm{PEO}$ & $\mathrm{LCP} / \mathrm{PEO}$ & PEO \\
\hline Crystal size $(\mu \mathrm{m})$ & $\sim 5$ & $5-10$ & $\sim 20$ & $\sim 20$ & $10-15$ \\
\hline Sample-to-detector distance (mm) & 550 & 300 & 300 & 300 & 400 \\
\hline Average flow rate $\left(\mathrm{nl} \mathrm{min}{ }^{-1}\right)$ & 56 & 17 & 182 & 155 & 79 \\
\hline Average crystal velocity $\left(\mu \mathrm{m} \mathrm{s}^{-1}\right)$ & 570 & 120 & 1550 & 1315 & 675 \\
\hline Average exposure time/crystal (ms) & 18.0 & 85.0 & 6.5 & 7.6 & 15.0 \\
\hline Maximum dose per crystal (MGy) & 0.1 & 0.1 & 0.1 & 0.1 & 0.1 \\
\hline Data-collection time $(\mathrm{h})$ & $\sim 14$ & 12 & $\sim 5$ & 2.5 & $\sim 3$ \\
\hline Protein/carrier volume $(\mu \mathrm{l})$ & 52.3 & 26.0 & 60 & 27.5 & 13.0 \\
\hline Wavelength $(\AA)$ & 1.03 & 1.03 & 1.03 & 1.03 & 1.03 \\
\hline Maximum resolution observed $(\AA)$ & 3.1 & 2.0 & 2.5 & 1.6 & 2.2 \\
\hline Resolution $(\AA)$ & $45-3.20(3.28-3.20)$ & $35-2.05(2.10-2.05)$ & $50-3.10(3.18-3.10)$ & $36.8-3.00(3.08-3.00)$ & $50-2.65(2.72-2.65)$ \\
\hline Space group & $C 222_{1}$ & $P 4_{3} 2_{1} 2$ & $H 32$ & $P 2_{1}$ & $P 4_{3} 2_{1} 2$ \\
\hline$a, b, c(\AA)$ & $39.4,179.5,140.3$ & $79.1,79.1,38.0$ & $186.4,186.4,60.3$ & $30.3,52.3,36.9$ & $68.3,68.3,108.2$ \\
\hline$\alpha, \beta, \gamma\left(^{\circ}\right)$ & $90,90,90$ & $90,90,90$ & $90,90,120$ & $90,92,90$ & $90,90,90$ \\
\hline No. of collected images & 503006 & 364724 & 159809 & 115672 & 97772 \\
\hline No. of hits/indexed patterns & $14711 / 5287$ & $124800 / 18648$ & $7912 / 1826$ & $13383 / 3157$ & $4497 / 817$ \\
\hline$\langle I / \sigma(I)\rangle$ & $7.7(0.3)$ & $11.2(0.4)$ & $7.6(1.9)$ & $5.1(2.5)$ & $4.6(0.3)$ \\
\hline Multiplicity & $142.6(33.2)$ & $873.3(43.9)$ & $156.4(4.8)$ & $45.1(13.0)$ & $104.8(40.6)$ \\
\hline Completeness (\%) & 99.8 & 99.8 & 99.7 & 100 & 99.2 \\
\hline $\mathrm{CC}^{*}(\%)$ & $0.992(0.423)$ & $0.978(0.436)$ & $0.986(0.799)$ & $0.973(0.856)$ & $0.954(0.431)$ \\
\hline$R_{\text {split }}(\%)$ & $13.4(506.0)$ & $10.7(242.8)$ & $14.2(73.09)$ & $25.9(38.8)$ & $22.4(331.0)$ \\
\hline
\end{tabular}

crystal density, corresponding to an average jet velocity of between 120 and $1250 \mu \mathrm{m} \mathrm{s}^{-1}$ in the $50 \mu \mathrm{m}$ diameter nozzle. Thus, we estimated that the exposure time of a single microcrystal across the $10 \mu \mathrm{m}$ X-ray beam was between $8 \mathrm{~ms}$ (for a flow rate of $182 \mathrm{nl} \mathrm{min}^{-1}$ ) and $85 \mathrm{~ms}$ (for a flow rate of $17 \mathrm{nl} \min ^{-1}$ ).

\subsection{Data processing, model building and refinement}

Data analysis is one of the major challenges of the serial crystallography approach owing to the serial nature of data collection from randomly oriented crystals of varying size and quality, the snapshot diffraction patterns of which consist of reflections measured with unknown partiality. In addition, the data collected during an experiment consist of actual crystal diffraction patterns and empty patterns (no crystals in the beam), resulting in the generation of terabytes of data. In order to monitor the hit rate and quality of large amounts of data in real time, we developed a real-time Python-based hit finder at APS. This program uses multiple processors to perform background subtraction and basic spot finding on each image as soon as it is transferred from the detector. Data reduction was also performed offsite using a modified version of Cheetah (Barty et al., 2014), called Cheetah-cbf, designed to read raw cbf images from the PILATUS detector and identify crystal diffraction patterns (hits) in HDF5 format, the suitable format for data processing with CrystFEL. Cheetah-cbf was installed on the GM/CA 23-ID-D beamline; it is accessible to all APS users and can also be used offsite. For each sample the peak-finding thresholds were carefully tailored to maximize the number of crystal hits and the number of Bragg peaks in each diffraction pattern.

Sorted diffraction patterns identified during the hit-finding process were subjected to indexing and structure-factor integration using CrystFEL v.0.6.2 (White et al., 2012, 2013, 2016). CrystFEL contains widely used algorithms such as MOSFLM (Powell et al., 2013), DirAx (Duisenberg, 1992) and $X D S$ (Kabsch, 2010), and a new algorithm known as asdf, which was recently implemented in CrystFEL. The new indexing option called 'retry', which was recently implemented in CrystFEL (White et al., 2016), allowed a significant increase in the number of successfully indexed patterns. This new option rejects a small fraction of the weakest spots and retries indexing. After each pattern was indexed, the intensities were merged and integrated using a Monte Carlo algorithm (Kirian et al., 2010, Kirian et al., 2011). Data-collection statistics for all of the proteins tested in this study are summarized in Table 1. The resolution-cutoff criteria were based on signal-to-noise ratios, completeness and correlation coefficients. The maximum radiation dose per crystal was estimated using the RADDOSE-3D server (Zeldin, Brockhauser et al., 2013; Zeldin, Gerstel et al., 2013), assuming cuboid crystals for all four crystal dimensions tested in this study $(5 \times 5 \times 5,10 \times 10 \times 10,15 \times 15 \times 15$ and $20 \times 20 \times$ $20 \mu \mathrm{m})$, a $10 \mu \mathrm{m}$ beamsize, a photon flux of $3.0-4.1 \times$ $10^{11}$ photons s$^{-1}$, an energy of $12 \mathrm{keV}$ and exposure times of 6.5 and $85 \mathrm{~ms}$, as described above. The results of this analysis are summarized in Table 1.

After integration with CrystFEL, the initial phases were obtained by molecular replacement with MOLREP (Vagin \& Teplyakov, 2010) using known structures of the proteins from the PDB [the PDB entries used were $5 \mathrm{k} 2 \mathrm{c}$ for $\mathrm{A}_{2 \mathrm{~A}} \mathrm{AR}$ (Batyuk et al., 2016), 4ziz for PC (Fromme et al., 2015), 4zix for lysozyme (Fromme et al., 2015), 5avj for proteinase K (Yazawa et al., 2016) and 2mu4 for Flpp3 (Zook et al., 2015)]. Additional remodeling performed by the $M R \_$protocol algorithm in Rosetta (Terwilliger et al., 2012) was required to obtain a sufficient phasing solution for Flpp3: loops from the published 
Table 2

SMX data-refinement statistics.

\begin{tabular}{|c|c|c|c|c|}
\hline & $\mathrm{A}_{2 \mathrm{~A}} \mathrm{AR}$ & Lysozyme & Phycocyanin & Proteinase K \\
\hline Total reflections & 25266 & 29412 & 17002 & 29685 \\
\hline No. of reflections used in refinement & 7702 & 7164 & 6280 & 5294 \\
\hline$R_{\text {work }} / R_{\text {free }}(\%)$ & $24.8 / 28.6$ & $22.8 / 26.8$ & $17.2 / 20.5$ & $22.5 / 24.3$ \\
\hline Protein & 2989 & 1002 & 2499 & 2032 \\
\hline Water and others (ligands or ions) & 151 & 21 & 127 & 9 \\
\hline Average $B$ value $\left(\AA^{2}\right)$ & 109.2 & 34.9 & 48.2 & 61.3 \\
\hline \multicolumn{5}{|l|}{ R.m.s. deviations from ideal values } \\
\hline \multicolumn{5}{|l|}{ Ramachandran plot statistics (\%) } \\
\hline Favored & 98.9 & 97.6 & 98.8 & 97.1 \\
\hline Allowed & 1.1 & 2.4 & 1.2 & 2.5 \\
\hline Disallowed & 0 & 0 & 0 & 0.4 \\
\hline Rotamer outliers & 0 & 1 & 2 & 0 \\
\hline PDB code & 5 uvi & 5uvj & 5 uvk & 5uvl \\
\hline
\end{tabular}

NMR structure (PDB entry 2mu4) were removed and then remodeled, with a total of 1000 new models, using fragments generated from the Rosetta webserver and initial phases provided by Phaser (McCoy et al., 2007). The ten lowest energy models were then resubmitted to Phaser, with several sufficient solutions obtained. The best model had an LLG score of 56.8 and a TFZ of 7.2. A model was built using the AutoBuilder protocol in PHENIX (Terwilliger et al., 2008), and the model was rephased for a final solution with an LLG of 300.7 and a TFZ of 14.7. Water molecules and ligands were removed from the reference structures for the phasing step. Structure refinement was carried out through multiple iterations of REFMAC5 (Murshudov et al., 2011) to refine atomic coordinates and isotropic $B$ factors. Manual inspection of the structures was carried out using Coot (Emsley \& Cowtan, 2004) after each refinement step. The figures were prepared with PyMOL (Schrödinger). Data-refinement statistics for all structures solved in this study are summarized in Table 2. Electron-density maps were calculated with the MAPS tool in the PHENIX software suite (Adams et al., 2010). Validation of all structures was carried out with the validation tools in the PHENIX software suite (Adams et al., 2010).

\section{Results and discussions}

Here, we demonstrate that the LCP injector-based SMX method is feasible at the GM/CA 23-ID-D beamline and can be used to determine the structures of a range of proteins of different sizes and types from crystal sizes between 5 and $20 \mu \mathrm{m}$. In addition, we introduce PEO gel as a novel carrier medium to deliver microcrystals into the X-ray beam, which can be utilized in serial crystallography experiments at synchrotron sources and XFELs. For our study, we used the high-viscosity injector developed at Arizona State University (Weierstall et al., 2014) to deliver microcrystals into the $10 \mu \mathrm{m}$ $\mathrm{X}$-ray beam path. Diffraction data were collected in a continuous shutterless mode using a PILATUS3 6M detector operating at $10 \mathrm{~Hz}$. The experimental setup at the beamline is shown in Fig. 2. To validate the proof-of-principle of the SMX approach, we chose a set of six proteins that includes one membrane protein, four soluble proteins and one multiprotein cofactor complex of different sizes as model systems: $\mathrm{A}_{2 \mathrm{~A}} \mathrm{AR}$, Flpp3, proteinase K, lysozyme, thaumatin and PC. Data from thaumatin microcrystals embedded in LCP were collected with a hit rate of $6 \%$. However, the number of indexed patterns was insufficient to yield a complete data set for this protein. Complete data sets were obtained from microcrystals of two proteins embedded in $\mathrm{LCP}\left(\mathrm{A}_{2 \mathrm{~A}} \mathrm{AR}\right.$ and lysozyme $)$ and three proteins in PEO gel (PC, Flpp3 and proteinase $\mathrm{K}$ ), which are described in further detail below.

\subsection{Protein structures in LCP viscous medium}

3.1.1. Lysozyme. Lysozyme was chosen as a test protein to evaluate the SMX method. Lysozyme was first crystallized and pelleted and was then reconstituted into LCP as described previously (Fromme et al., 2015; Liu et al., 2014). The resulting microcrystal-LCP suspension, containing lysozyme crystals of between 5 and $10 \mu \mathrm{m}$ in size, was then transferred to the LCP injector and diffraction data were collected using an LCP flow

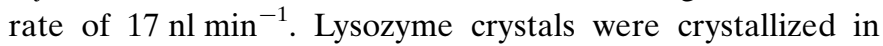
space group $\mathrm{P}_{3} 2_{1} 2$ and were occasionally seen to diffract to beyond $2.0 \AA$ resolution (Supplementary Fig. S3). A total of 364724 images were collected, of which 124800 were classified as hits (hit rate $34.2 \%$ ) and 18648 were successfully indexed. The final data-collection statistics are given in Table 1 . The structure of lysozyme was solved by molecular replacement with PDB entry 4zix (Fromme et al., 2015) as a search model without waters or ions. The structure was refined at a resolution of $2.05 \AA$ with an $R_{\text {work }}$ and $R_{\text {free }}$ of 22.8 and $26.8 \%$, respectively (Table 1 ). The final refinement statistics are given in Table 2. The resulting experimental maps were of excellent quality, revealing the presence of 18 water molecules, three chloride ions and a sodium ion. The sodium ion shows an octahedral coordination by four neighboring residues and two water molecules (Fig. 3).

To ensure the validity of our procedure, the structure determined in our study was compared with those obtained by the serial method at XFELs using an LCP injector (Fromme et 
al., 2015) and a GDVN injector (Boutet et al., 2012), and at synchrotron sources using a high-viscosity injector (Botha et al., 2015), glass capillaries (Stellato et al., 2014) and cryocooled crystals on a fixed target (Murray et al., 2015). All structures superimposed very well, with root-mean-square deviation (r.m.s.d.) values of $\sim 0.4 \AA$ for $\mathrm{C}^{\alpha}$ atoms $(\sim 0.8 \AA$ for all atoms of the protein). Only small differences were detected at the side chains of highly solvent-exposed residues, which can be explained in terms of the variation in crystal-preparation protocols and crystal-delivery methods. Analysis of the $B$ factor distribution revealed no significant differences overall between our structure $\left(35 \AA^{2}\right)$ and the cryocooled structure (31 $\AA^{2}$; Murray et al., 2015) and those collected at XFELs using the GDVN injector (35 $\AA^{2}$; Boutet et al., 2012) and the LCP injector (27 $\AA^{2}$; Fromme et al., 2015). In addition, lower $B$ factors are observed for our structure compared with those determined using glass capillaries (52 $\AA^{2}$; Stellato et al., 2014) and the high-viscosity injector of Botha and coworkers (48 $\AA^{2}$; Botha et al., 2015) .

It has been suggested that at room temperature the radiation-dose limit above which radiation damage becomes significant is 0.3 MGy (Nave \& Garman, 2005). In a recent study carried out by Coquelle and coworkers in which a roomtemperature raster-scanning serial crystallography method was used at the ESRF synchrotron, the maximum dose per crystal was estimated to be between 3.2 and 29.1 MGy (Coquelle et al., 2015). They demonstrated that mainly the S atoms in lysozyme and, in particular, disulfide bonds were damaged by radiation to some extent, although other structural information was not compromised (Coquelle et al., 2015). In our study the average maximum radiation dose per crystal was estimated to be $0.1 \mathrm{MGy}$ (Table 1), which is below the dose beyond which radiation damage is expected. In order to assess whether the disulfide bonds of our lysozyme structure were radiation-damaged considering the above radiation dose, we calculated the structure-factor amplitude Fourier difference $\left(F_{\mathrm{o}}-F_{\mathrm{o}}\right)$ maps between our data set and the data set collected using the LCP injector at the LCLS (Fromme et al., 2015). The maximum radiation dose per crystal for the SFX data was $2.5 \mathrm{MGy}$, which is much higher than the maximum dose limit of 0.3 MGy (Nave \& Garman, 2005). As shown in Fig. 4, none of the four disulfide bonds in lysozyme, including the disulfide bridge Cys64-Cys80, which was considered to be the most radiation-sensitive at room temperature by Coquelle and coworkers, show negative peaks at the $\mathrm{S}$ atoms or at the disulfide bridges. In addition, radiation damage was not visible in the $2 m F_{\mathrm{o}}-F_{\mathrm{o}}$ and $m F_{\mathrm{o}}-F_{\mathrm{o}}$ maps (Fig. 4), indicating that the disulfide bonds were not broken and the structural information has not been compromised.

3.1.2. $A_{2 A} A R$. Microcrystals of $A_{2 A} A R$ were injected into the $\mathrm{X}$-ray beam path at an average flow rate of $56 \mathrm{nl} \mathrm{min}-1$ and measured for $\sim 14 \mathrm{~h}$. Less than $55 \mu \mathrm{l}$ of LCP-sample was necessary to collect a total of 500003 images, of which 14711 were identified as hits (3\% hit rate) and 5287 (indexing yield $36 \%$ ) were successfully indexed in space group $C 222_{1}$ (Table 1). Fig. 5 shows a diffraction pattern of a single $A_{2 A} A R$ microcrystal. The structure of $\mathrm{A}_{2 \mathrm{~A}} \mathrm{AR}$ was solved by molecular replacement using the recently reported highresolution SFX structure (PDB entry 5k2c; Batyuk et al., 2016) without waters and ligands. The final structure was refined to $3.2 \AA$ resolution with an $R_{\text {work }}$ and $R_{\text {free }}$ of 24.2 and $28.6 \%$, respectively (Table 1$)$. The final data-collection and refinement statistics are given in Tables 1 and 2. Despite the medium resolution of the structure, the high quality of the electrondensity maps allowed us to model the ligand ZM241385, three cholesterol molecules and three other lipids (Fig. 6). However, the clusters of water molecules previously described in the structures determined by SFX and under cryoconditions, which were demonstrated to play a key role in maintaining the stability of the receptor and in ligand binding, were not identified in our medium-resolution $\mathrm{A}_{2 \mathrm{~A}} \mathrm{AR}$ structure. Also, the sodium ion which has been described to play a key role in the receptor-activation mechanism (Katritch et al., 2014) was not identified in our structure, possibly for the same reason.

We compared the structure of $\mathrm{A}_{2 \mathrm{~A}} \mathrm{AR}$ obtained in our study with high-resolution structures obtained by SFX (Batyuk et al., 2016) and from cryocooled crystals (Liu et al., 2012). All structures aligned very closely, with r.m.s.d. values for $\mathrm{C}^{\alpha}$ atoms of 0.3 and $0.4 \AA$ for the SFX and synchrotron structures, respectively. Larger differences were found around the side chains of solvent-exposed bulky residues, the orientation of which could not be well modeled. The discrepancies were slightly larger in the BRIL fusion protein, where small differences were even observed along the backbone of this protein. The average $B$ factors were also significantly higher (109 $\AA^{2}$ for the whole protein, $148 \AA^{2}$ for the BRIL fragment and $96 \AA^{2}$ for the $\mathrm{A}_{2 \mathrm{~A}} \mathrm{AR}$ fragment) compared with that of the room-temperature structure solved by SFX $\left(\sim 40 \AA^{2}\right.$ for the whole protein; Batyuk et al., 2016). Also, the observed high $B$ factors may explain why no water molecules are seen in our structure. High average $B$ factors $\left(90-100 \AA^{2}\right.$ ) have so far been reported in the literature for 86 structures deposited in the

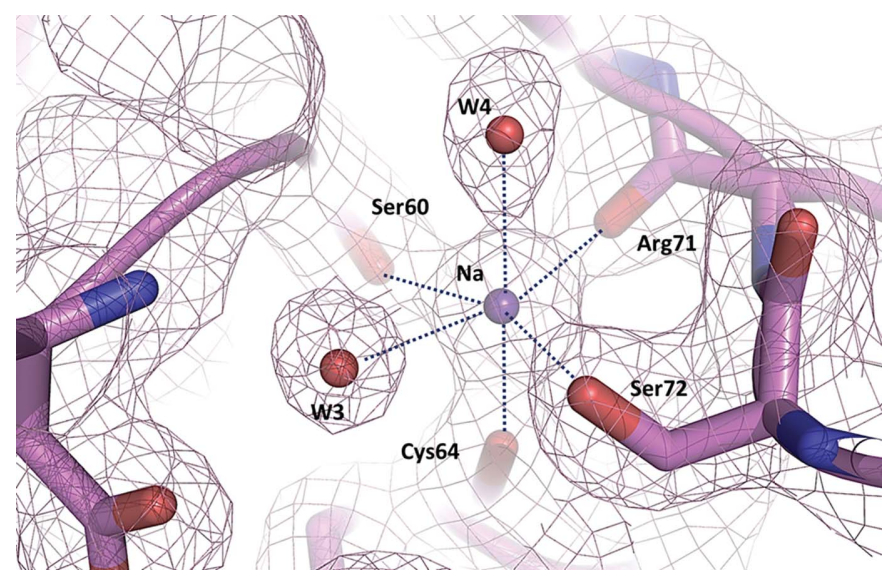

Figure 3

Quality of the electron-density maps displayed around the octahedral coordination of sodium in the lysozyme structure. $2 m F_{\mathrm{o}}-D F_{\mathrm{c}}$ electrondensity maps are shown as light pink mesh contoured at $1 \sigma$. The residues coordinating the $\mathrm{Na}$ atom (Ser60, Cys64, Arg71 and Ser72) are shown as a magenta stick representation. Waters W3 and W4 are shown as red spheres. The interactions between sodium and its ligands are represented as blue dotted lines. 
PDB at resolutions between 3.15 and $3.25 \AA$. In fact, high $B$ factors should be expected when solving medium- to lowresolution crystallographic structures at room temperature.

The $\mathrm{A}_{2 \mathrm{~A}} \mathrm{AR}$ protein has $15 \mathrm{Cys}$ residues in its sequence, eight of which form four disulfide bonds (Cys71-Cys159, Cys74-Cys146, Cys77-Cys166 and Cys259-Cys262), which might be subject to radiation damage. In order to assess the effect of radiation damage, we calculated the structure-factor amplitude Fourier difference $\left(F_{\mathrm{o}}-F_{\mathrm{o}}\right)$ maps between our data set and the undamaged data set collected at the LCLS (Batyuk et al., 2016). As shown in Supplementary Fig. S4, the
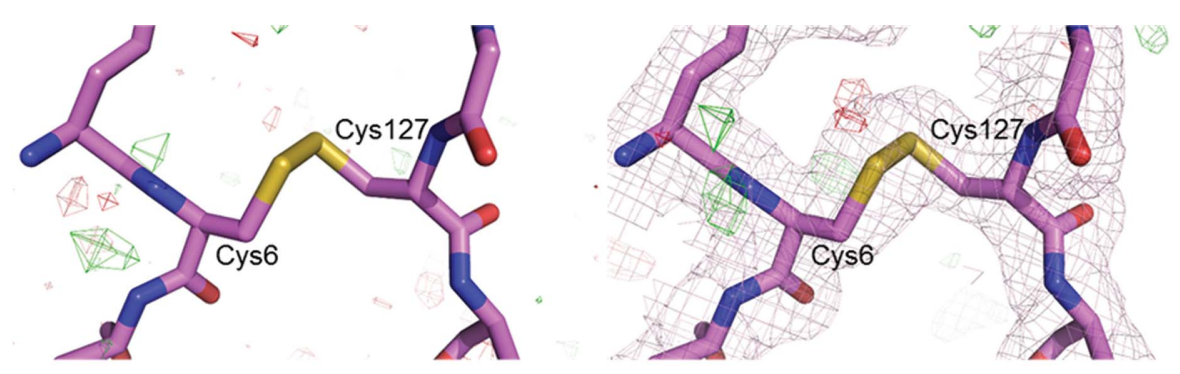

(a)

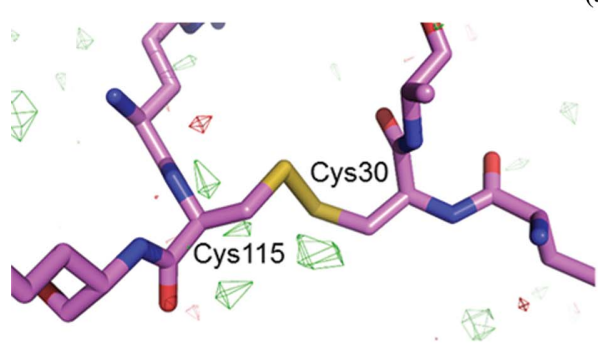

(a)

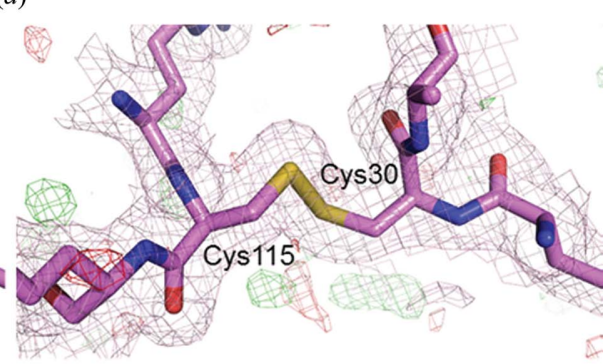

(b)

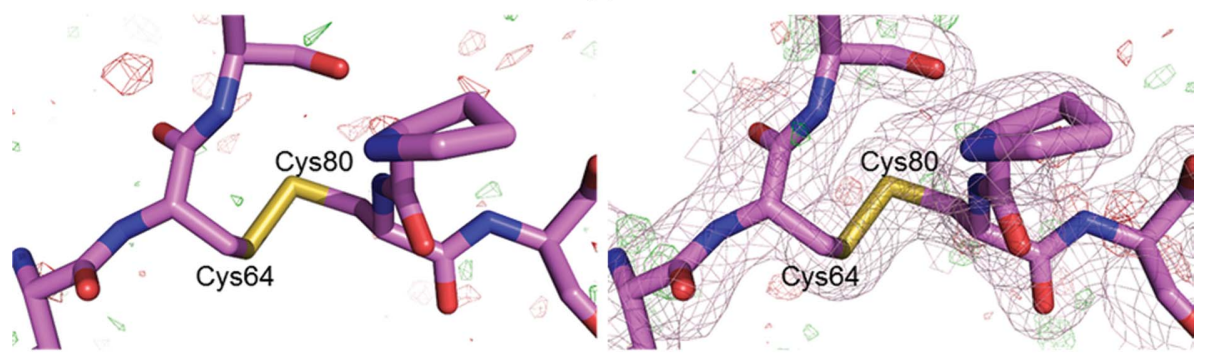

(c)
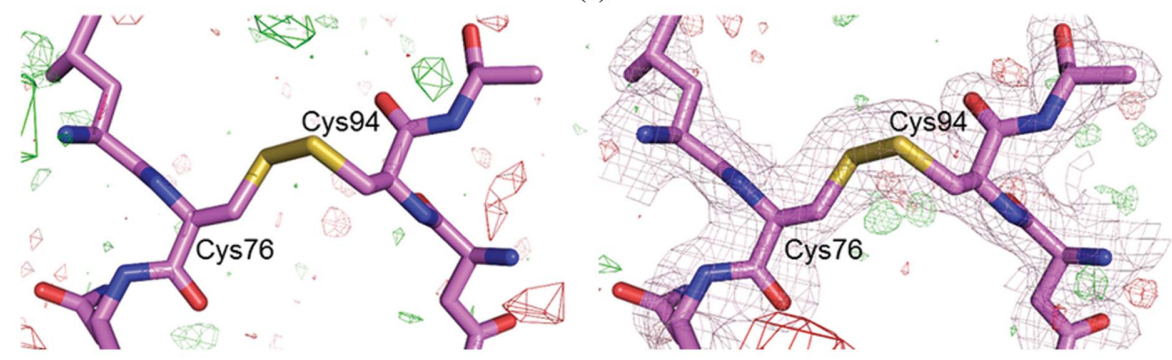

(d)

Figure 4

Electron-density maps displayed around the four disulfide bridges in lysozyme: (a) Cys6-Cys127, (b) Cys30-Cys115, (c) Cys64-Cys80 and (d) Cys76-Cys94. Cysteines and neighboring residues are represented as pink sticks. Left panels, structure-factor amplitude Fourier difference $\left(F_{\mathrm{o}}-F_{\mathrm{o}}\right)$ maps at $3 \sigma$ between our data and the data set collected at LCLS (Fromme et al., 2015), with red and green contours indicating negative and positive density, respectively. Right panels, $2 m F_{\mathrm{o}}-D F_{\mathrm{c}}$ (light pink mesh, contoured at $1 \sigma$ ) and $m F_{\mathrm{o}}-D F_{\mathrm{c}}$ (red and green meshes, contoured at $3 \sigma$ ) maps. absence of negative electron-density peaks around disulfide bonds indicates that the radiation-damage effect is limited, as expected by the estimated radiation dose of $0.2 \mathrm{MGy}$. The $2 m F_{\mathrm{o}}-F_{\mathrm{o}}$ and $m F_{\mathrm{o}}-F_{\mathrm{o}}$ electron-density maps also demonstrate that the disulfide bonds are not broken (Supplementary Fig. S4).

\subsection{Protein structures in the novel PEO viscous medium}

3.2.1. Flpp3. A highly concentrated microcrystal pellet was mixed with either LCP or PEO gel. The LCP- and PEOembedded microcrystals were transferred to the high-viscosity injector (Weierstall et al., 2014) and presented into the beam in a free-flowing stream. Diffraction data for the Flpp3 microcrystals were collected at an average flow rate of $155 \mathrm{nl} \mathrm{min}{ }^{-1}$, consuming less than $30 \mu \mathrm{l}$ of protein/carrier sample, which yielded 13383 hits, of which 3157 were indexed at $3.0 \AA$ resolution (Table 1). The final data set was generated from reflections collected from both PEO and LCP samples. Efforts to generate a sufficient model for Flpp3 at 3.0 ^ resolution are ongoing. The final data-collection and refinement statistics for the Flpp3 data set are shown in Tables 1 and 2 .

3.2.2. Phycocyanin (PC). The PEO with embedded PC microcrystal ( $\sim 20 \mu \mathrm{m})$ suspension was injected at an average flow rate of $182 \mathrm{nl} \mathrm{min}^{-1}$ and 159809 images were collected, which correspond to an effective measurement time of $\sim 5 \mathrm{~h}$ (Table 1). This resulted in a PC sample consumption of about $60 \mu \mathrm{l}$. The structure of PC was determined to $3.1 \AA$ resolution from 1826 indexed patterns (indexing rate $23 \%$; Table 1 ). The final data-collection and refinement statistics of the PC data set are shown in Tables 1 and 2. Supplementary Fig. S5 shows a diffraction pattern of a single PC-PEO microcrystal. Slightly smeared Bragg peaks were observed, which indicates that the PC crystals were rotating azimuthally around the X-ray beam axis during the $100 \mathrm{~ms}$ exposure. This has previously been observed for lysozyme microcrystals embedded in LCP with $100 \mathrm{~ms}$ exposures (Botha et al., 2015). The structure of PC was solved by molecular replacement using the previously solved LCP-injected SFX structure as a search model, with water molecules and chromophores removed 


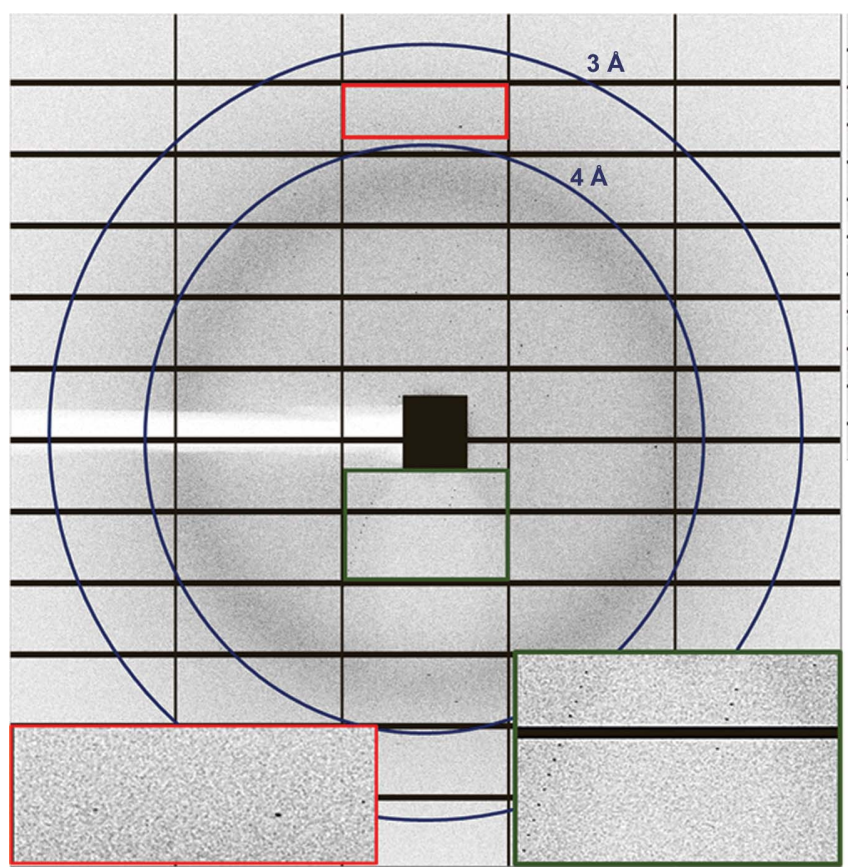

(a)

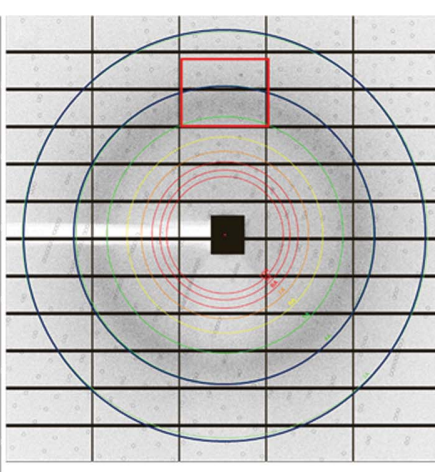

(b)

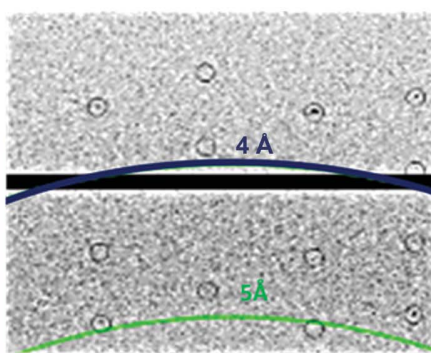

(c)
Figure 5

Diffraction pattern of a single microcrystal of $\mathrm{A}_{2 \mathrm{~A}} \mathrm{AR}$ in LCP. (a) Raw diffraction pattern with resolution rings at $3 \AA$ and $4 \AA$ showing visible Bragg spots extending to about $3.5 \AA$ resolution (red inset panel). The green inset panel shows low-resolution Bragg spots. $(b)$ The same diffraction pattern as in $(a)$ after indexing with resolution rings. (c) Closer view of the red boxed area highlighted in $(b)$.

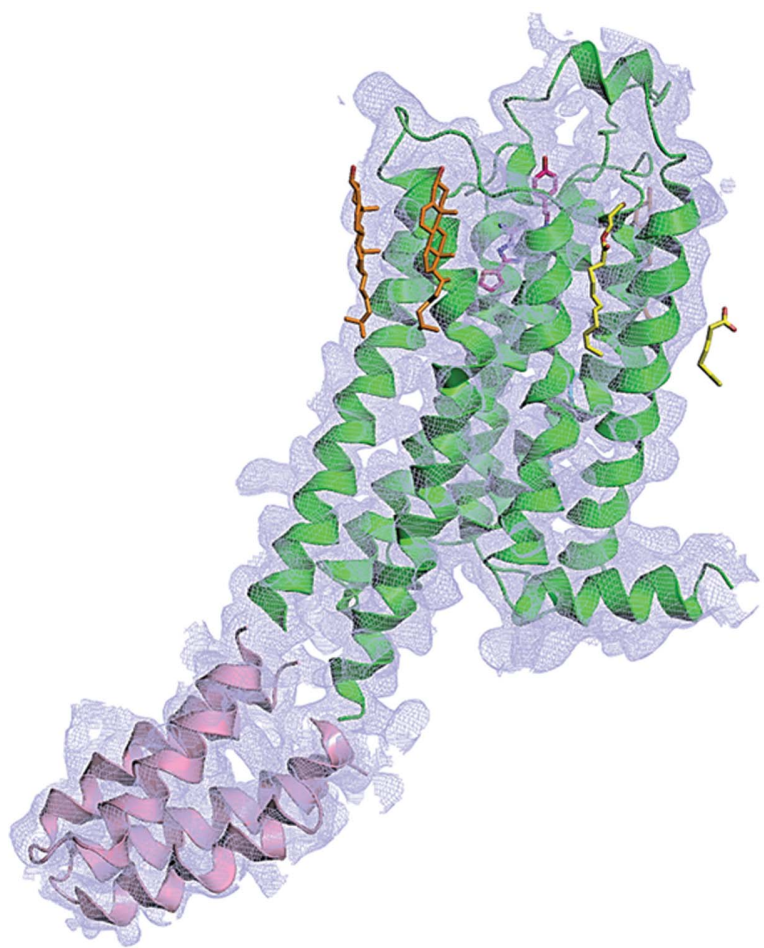

(a)

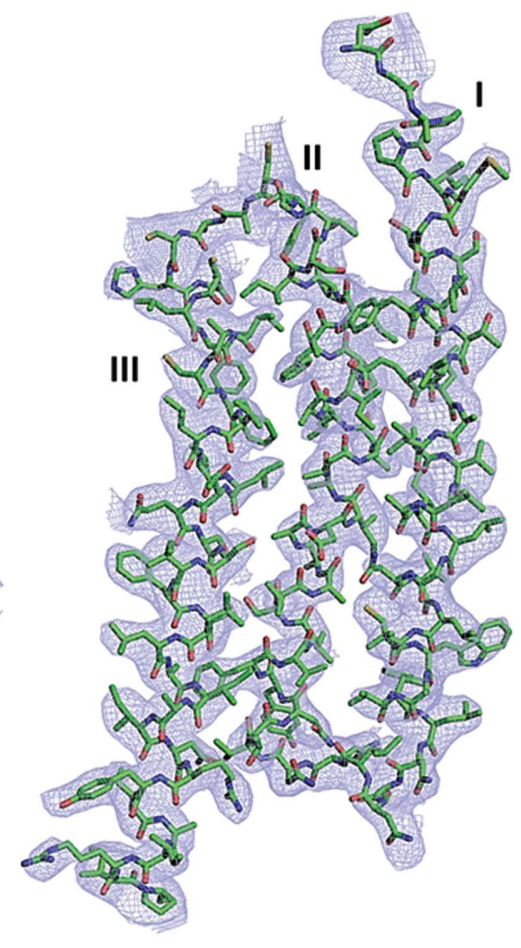

(b)
Figure 6

The quality of the $2 m F_{\mathrm{o}}-D F_{\mathrm{c}}$ electron-density maps reflects the good quality of the collected data. (a) The $\mathrm{A}_{2 \mathrm{~A}} \mathrm{AR}$ protein is shown as a green cartoon and the BRIL fusion protein is shown as a pink cartoon. The ligand ZM241385 (magenta), three cholesterols (orange) and three lipids (yellow and cyan) are shown as stick representations. $(b) 2 m F_{\mathrm{o}}-D F_{\mathrm{c}}$ electron-density maps around the residues, shown as sticks, for helices I, II and II.
(PDB entry 4ziz; Fromme et al., 2015). The PC structure was refined to $3.1 \AA$ resolution, with final $R_{\text {work }}$ and $R_{\text {free }}$ values of 17.2 and $20.4 \%$, respectively. The quality of the structure can be assessed from the $2 m F_{\mathrm{o}}-D F_{\mathrm{c}}$ electron-density maps of the three chromophores of PC (Fig. 7a). To further evaluate the quality of our structure, we compared our model with the two SFX structures recently described using the LCP injector (PDB entry 4ziz; Fromme et al., 2015) and the GDVN injector (PDB entry 4q70; R. Fromme, S. Roy-Chowdhury, S. Basu, C. Yoon, D. Brune \& P. Fromme, unpublished work). The three structures aligned very closely with each other, with r.m.s.d. values for $\mathrm{C}^{\alpha}$ atoms of 0.1 and $0.7 \AA$ for the LCP and GDVN structures, respectively. Furthermore, we compared the PC structure with the structure previously solved at a synchrotron using a single cryocooled crystal (PDB entry 310f; R. Fromme, D. Brune \& P. Fromme, unpublished work). Both structures superimposed very well, with an r.m.s.d. value of $0.2 \AA$ for all $\mathrm{C}^{\alpha}$ atoms. An r.m.s.d. value of $0.5 \AA$ for all atoms indicates that small differences were, however, observed in the loop regions and in the solvent-exposed regions. The average $B$ factors for both chains of PC ( $45 \AA^{2}$ for chain $A$ and $51 \AA^{2}$ for chain $B$ ) showed no significant differences from the LCP structure (38 $\AA^{2}$ for chain $A$ and $45 \AA^{2}$ for chain $B$ ). However, higher average $B$ factors were observed compared with those of the cryocooled ( $21 \AA^{2}$ for chain $A$ and $25 \AA^{2}$ for chain $B$ ) and the GDVN (26 $\AA^{2}$ for chain $A$ and $32 \AA^{2}$ for chain $B$ ) structures. These differences are attributable, to a greater extent, to the higher resolution of the structures collected at cryogenic temperatures $\quad\left(1.35 \AA^{2} ; \quad\right.$ R. Fromme, D. Brune \& P. Fromme, unpublished work) and using a 
liquid jet (1.9 $\AA^{2}$; R. Fromme, S. Roy-Chowdhury, S. Basu, C. Yoon, D. Brune \& P. Fromme, unpublished work).

3.2.3. Proteinase K. Diffraction data for proteinase K (PK) microcrystals embedded in PEO gel were collected at a constant flow rate of $79 \mathrm{nl} \mathrm{min}^{-1}$ with an effective measurement time of $\sim 3 \mathrm{~h}$, during which nearly 100000 images were recorded. This resulted in a PK sample consumption of less than $15 \mu \mathrm{l}$. A total of 4497 images with diffraction to a maximum resolution of $2.2 \AA$ were identified as hits (hit rate $4.2 \%$ ), of which 817 were successfully indexed in space group $P_{3} 2_{1} 2$ (Table 1). The final data-collection and refinement statistics are given in Tables 1 and 2. Supplementary Fig. S6 shows a diffraction pattern from a single proteinase K-PEO microcrystal. As with PC, slightly smeared Bragg peaks were also observed, which indicates that the proteinase $\mathrm{K}$ crystals were rotating during the $100 \mathrm{~ms}$ exposure. Phasing was performed by molecular replacement using the cryocooled structure at a synchrotron (PDB entry 5avj; Yazawa et al., 2016) as a search model. The final refinement gave an $R_{\text {work }}$ and $R_{\text {free }}$ of 22.5 and $24.3 \%$, respectively. The proteinase $\mathrm{K}$ structure was determined to $2.65 \AA$ resolution and, overall, our structure is very similar to that solved using cryocooled crystals, with an r.m.s.d. value of less than $0.1 \AA$ for all $\mathrm{C}^{\alpha}$ atoms. The average $B$ factor was $61 \AA^{2}$, as expected for a structure solved to $2.65 \AA$ resolution at room temperature. In our structure, no water molecules were identified; however, the two $\mathrm{Ca}$ atoms that contribute to protein stability were clearly visible. The $2 m F_{\mathrm{o}}-D F_{\mathrm{c}}$ electron-density maps around the one of these two Ca atoms can be used to assess the high quality of our proteinase K structure (Fig. 7b).
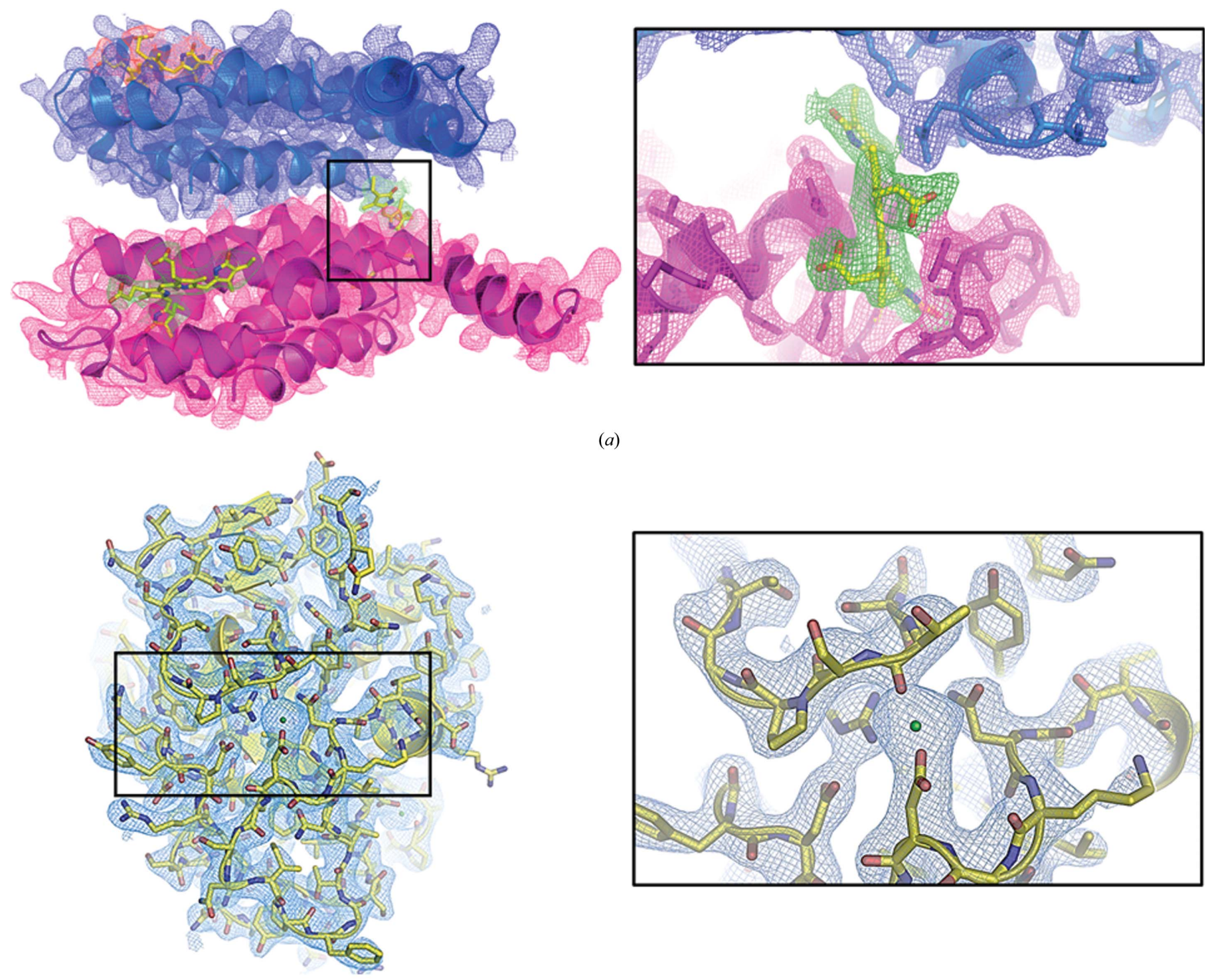

(a)

(b)

Figure 7

$2 m F_{\mathrm{o}}-D F_{\mathrm{c}}$ electron-density maps of PC $(a)$ and proteinase $\mathrm{K}(b)$ contoured at $1 \sigma .(a)$ The two PC subunits $(\alpha$, blue; $\beta$, pink) are shown in cartoon representation. The three chromophores are shown as yellow sticks. A closer view of the chromophore in the black box is shown in the right panel. $(b)$ Proteinase $\mathrm{K}$ is shown in a yellow cartoon and stick representation. One of the two Ca atoms is shown as a green sphere. A closer view of the Ca atom is illustrated in the right panel. 
3.3. PEO gel: a novel high-viscosity medium as a crystal carrier for serial crystallography

One of the greatest limitations of SMX experiments at synchrotron sources using monochromatic X-rays is that the crystals have to be exposed for longer times, which decreases the data-acquisition rates at these facilities (typically $<20 \mathrm{~Hz}$ ) compared with those at XFELs $(120 \mathrm{~Hz}$ at LCLS and $60 \mathrm{~Hz}$ at SACLA). Delivering crystals in a liquid jet using the GDVN injector has been successfully used for many SFX experiments at XFELs (for a review, see Martin-Garcia et al., 2016). The GDVN injector operates at a minimal flow rate of $10 \mu \mathrm{min}^{-1}$, which is too fast even for the repetition rates of current XFELs; thus, most of the sample is wasted and large sample volumes are needed to obtain a complete data set. Sample waste is even much more noticeable at the low repetition rates at synchrotrons, which makes liquid injectors impractical. Additionally, with the high flow rate of the GDVN the crystal transit of the X-ray beam is submillisecond, resulting in very weak diffraction; therefore, the GDVN is not recommended for SMX experiments. New strategies have allowed crystals to be delivered into the X-ray beam path more efficiently so that sample waste is markedly reduced. One of these methods is the use of a high-viscosity medium extruded as a continuous stream using the LCP injector (Weierstall et al., 2014) at very slow flow rates (typically 1-300 $\mathrm{nl} \mathrm{min}^{-1}$ ), allowing extremely low sample consumption (less than $1 \mathrm{mg}$ ) compared with a GDVN injector (10-100 mg protein).

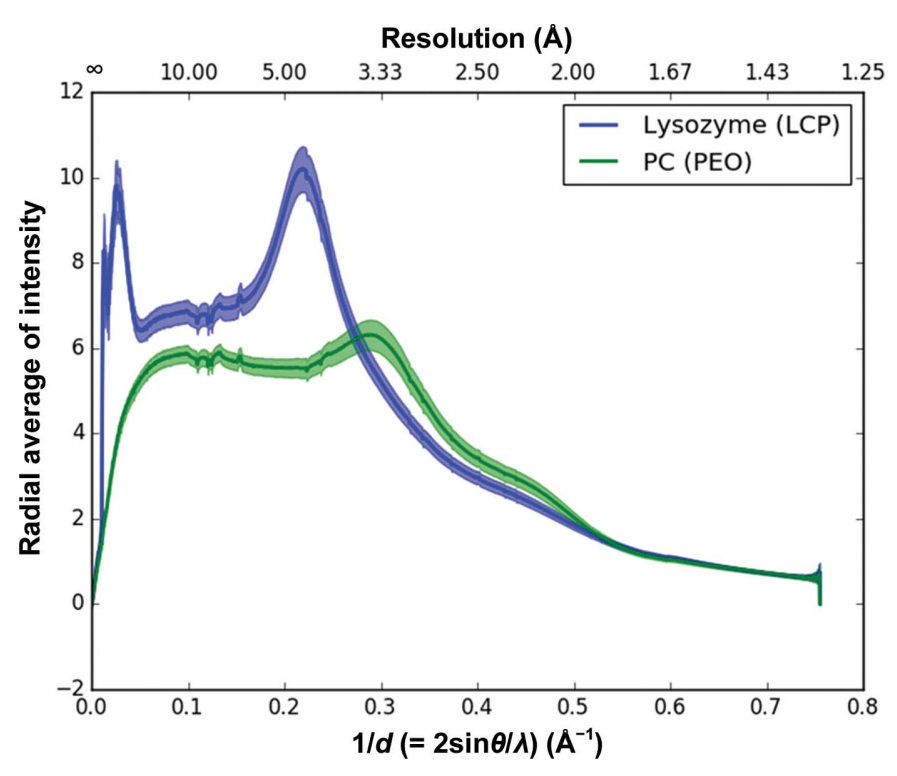

Figure 8

Diffuse background scattering comparison between PEO gel and LCP. The mean radial intensity over the total number of frames used for each medium protein is plotted against resolution $(1 / d)$. The cyan line represents the mean radial intensity for lysozyme in LCP medium as a function of $1 / d$ (or resolution in $\AA$ on the upper $x$ axis). The green line represents the mean radial intensity for PC in PEO gel medium as a function of $1 / d$. The error or fluctuation in the radial intensity is quantified using the mean absolute deviation of both media, which is shown as transparent regions. The lines end at different resolution points owing to the different crystal-to-detector distances.
In our study, we have used LCP as a crystal carrier medium which can also be used as a host matrix to crystallize membrane proteins (Caffrey \& Cherezov, 2009). To date, the use of LCP has led to the structure determination of a wide range of membrane proteins, including ion channels, transporters, enzymes, $\beta$-barrels and, in particular, GPCRs (Xiang et al., 2016). More recently, LCP has been successfully used to deliver previously grown soluble protein crystals into the $\mathrm{X}$-ray beam in serial crystallography experiments, allowing their structure determination using XFELs (Fromme et al., 2015) and synchrotrons (Botha et al., 2015; Nogly et al., 2015; Stellato et al., 2014). In the case of membrane proteins, crystals have to be grown in LCP since mixing membrane-protein crystals pregrown in solution in the form of protein-detergent micelles with LCP typically leads to dissolution of the crystals. This is probably owing to partitioning of detergent molecules into the lipid bilayer of LCP, leading to crystal dissolution and protein denaturation.

A novel high-viscosity medium has now been presented here to deliver protein microcrystals for SMX experiments. A gel polymer of high-molecular-weight poly(ethylene oxide) (PEO) has been used as a host matrix to embed previously grown protein microcrystals of Flpp3, proteinase K and PC. PEO has the same chemical composition as PEG, a commonly used precipitating agent in protein crystallography, but owing to its high molecular weight it is referred to by a different name. PEO is one of the most studied water-soluble synthetic polymers (Bailey, 1976; Hammouda et al., 2004) and is used as a model for biomedical applications such as in drug-delivery systems (Dhawan et al., 2005). PEO is soluble in water over a wide range of degrees of polymerization and at moderate temperatures $\left(20-30^{\circ} \mathrm{C}\right.$; Blank, 1974). The use of PEO as a gel has been reported for the crystallization of a wide variety of compounds from inorganic solids (Bianconi et al., 1991) to organic molecules (Chandrasekhar, 2000; Choquesillo-Lazarte \& García-Ruiz, 2011) and, recently, proteins (Pietras et al., 2010). In contrast to Mebiol, a medium that is viscous only at temperatures above $25^{\circ} \mathrm{C}$ and that has recently been used as a crystal carrier (Botha et al., 2015), PEO gels are highly stable at a wide range of temperatures, so that PEO-mediated crystal delivery can be accomplished at the traditional crystallization temperatures of $4-30^{\circ} \mathrm{C}$. Additionally, making PEO gel is a very simple and straightforward procedure, as described in $\$ 2$, leading to a highly homogeneous crystal distribution. PEO gels, like any other PEG, are compatible with a wide variety of precipitants commonly used in protein crystallization and have also been shown to be compatible with a wide variety of organic solvents that are commonly used as additives in many crystallization recipes (Choquesillo-Lazarte \& García-Ruiz, 2011). In all of our experiments, PEO-embedded crystals established very stable streams and no signs of dehydration were observed. Fig. 8 shows a comparison of the diffuse background X-ray scattering of the PEO gel stream compared with an LCP stream under the same experimental conditions. Details of the analysis used to evaluate background scattering is included in the Supporting Information. The characteristic broad peak at about $4.5 \AA$ resolution corresponding to diffuse 
scattering of the LCP stream can be seen in Fig. 8. Diffuse scattering from PEO gel is observed in the $3.3 \AA$ resolution region; it is mostly dominated by water scattering owing to the low PEO content. Overall, the background scattering from PEO gel medium is roughly 1.5 times less than that from LCP in the diffuse-ring regions (resolutions between 3.5 and $6 \AA$ ). In addition, unlike PEO gel, LCP shows strong scattering at resolutions of less than $30 \AA$, which makes PEO gel an ideal crystal carrier for protein crystals with large unit cells. A similar diffuse scattering profile to that of PEO gel has previously been reported for agarose medium (Conrad et al., 2015). This is not surprising considering that both PEO and agarose gels have a high water content (over 90\%) compared with that of LCP $(\sim 50 \%)$. Therefore, agarose and PEO have been demonstrated to be highly stable viscous media, and are compatible with a wide variety of crystallization compounds, making them suitable as crystal carriers.

Preliminary studies indicate that PEO gel is also suitable for mixing with crystals of membrane proteins such as photosystem I (PSI), a large membrane-protein complex of $1080 \mathrm{kDa}$ containing 36 protein subunits and more than 300 cofactors. Observation of PSI crystals immersed in PEO by ultraviolet two-photon excited fluorescence (UV-TPEF), polarized light and SONICC (Kissick et al., 2011) imaging indicate that PSI crystals remain intact after mixing with PEO (Supplementary Fig. S7).

\section{Conclusions and outlook}

Here, we present a proof-of-concept demonstration for the data collection of X-ray diffraction data sets suitable for structure determination from macromolecular, micrometresized crystals at room temperature using a high-viscosity injector on the GM/CA 23-ID-D beamline at APS. The number of crystallographic experiments carried out at room temperature has repaidly grown since the first SFX experiment was conducted at an XFEL in 2009 (Chapman et al., 2011). XFELs offer unique X-ray beam properties (ultrashort, extremely intense pulses with a high repetition rate and coherence), which enables the determination of roomtemperature structures, outrunning most radiation damage. However, the number of XFEL sources is limited, and therefore the structural biology community has begun to adapt the serial crystallography method at conventional synchrotron sources. Even though synchrotron sources are not as powerful as XFELs, they can produce sufficiently bright X-ray beams to enable data to be collected from 5-20 $\mu \mathrm{m}$ sized crystals on millisecond timescales. Also, recent and future developments and upgrades in optics, sample delivery, detector technology and synchrotrons themselves will make these radiation sources even more suitable for serial data collection. Synchrotron sources themselves are more accessible worldwide compared with XFELs. For all of these reasons, the number of SMX experiments is increasing rapidly (Botha et al., 2015; Coquelle et al., 2015; Gati et al., 2014; Hasegawa et al., 2017; Heymann et al., 2014; Huang et al., 2015,
2016; Murray et al., 2015; Nogly et al., 2015; Stellato et al., 2014; Zander et al., 2015).

The work presented here is an important step towards adapting the serial approach for room-temperature synchrotron data collection from a broad range of protein targets. The use of high-viscosity crystal carrier media coupled with a fastframing detector allowed us not only to synchronize a moving crystal through an intersecting X-ray beam with the desired exposure time, but also to considerably reduce sample consumption by a factor of 20 compared with SFX experiments with the GDVN injector. The utility of a high-viscosity stream for delivering crystals in the SMX approach has been demonstrated previously, including the structure determination of bacteriorhodopsin (bR) at the ESRF (Nogly et al., 2015) and the structure determination of lysozyme at SLS (Botha et al., 2015). High-viscosity injectors such as that used in this study (Weierstall et al., 2014) offer the possibility of using different viscous materials other than LCP as a crystal carrier medium. Embedding pregrown crystals in a high-viscosity medium may alter the properties of the matrix or even dissolve the crystals. Thus, the choice of the crystal carrier can be critical to the success of serial crystallography experiments. In our study, we have explored LCP medium as a crystal carrier medium and have shown that PEO gel is also suitable as a general delivery system for soluble proteins for experiments at synchrotrons as well as XFELs. Based on the visual techniques mentioned, PEO gels might also be suitable to deliver membrane-protein crystals in serial crystallography experiments.

Owing to the low flux density currently achievable at synchrotron beamlines compared with that of XFELs, we decided to use crystal sizes that varied from 5 to $20 \mu \mathrm{m}$, which allowed us to collect a complete SMX data set for four proteins by using no more than $60 \mu \mathrm{l}$ of protein-carrier sample. The current implementation of the serial synchrotron strategy will improve with the future upgrades at the APS, which will create a smaller, brighter microfocused beam with an intensity that is up to two orders of magnitude higher. These improvements, along with new developments in beamline optics and the acquisition of faster frame-readout detectors (e.g. EIGER), will allow X-ray structure determination from much smaller crystals, possibly on the submicrometre scale. These improvements will probably improve the resolution of, for example, crystals of $\mathrm{A}_{2 \mathrm{~A}} \mathrm{AR}$ using a similar crystal size $(\sim 5 \mu \mathrm{m})$ from the $3.2 \AA$ resolution obtained in this study to possibly better than $2.5 \AA$ resolution. Another interesting prospect for serial crystallography at synchrotron sources is to use a broad-bandpass beam such as that at the BioCARS beamline at the APS. Instead of using a monochromator, a mirror is used as a low-pass filter, creating a 'pink beam', which can be 500-fold more intense than a monochromated beam. Preliminary experiments at BioCARS have shown that serial crystallography data can be collected with a single 100 ps pulse when the APS is operating in hybrid mode or with four bunches $(460 \mathrm{~ns})$ in standard 24-bunch mode (MartinGarcia et al., in preparation). However, larger crystals were required than for this monochromatic study owing to the 
larger beam size at BioCARS compared with GM/CA. The APS-U will allow the pink beam to be focused more tightly, enabling serial crystallography on microcrystals. The wider bandwidth of the pink beam compared with a monochromatic beam enables the measurement of full reflections, which will significantly diminish the effect of partiality on the data (White et al., 2013) and lead to fewer required patterns for a complete data set.

The study presented here not only demonstrates the current feasibility of the serial crystallography method for determining static structures, but also suggests its extension to timeresolved pump-probe measurements to study light-induced reactions on millisecond timescales (or faster timescales when shorter exposure times are available) from microcrystals. Traditionally, time-resolved crystallography has been performed on large single crystals mounted on a goniometer by Laue crystallography using a pink beam (for a review, see Hajdu \& Johnson, 1990). Time-resolved studies at synchrotrons have always been limited by the pulse length achievable at these facilities. However, over the past twenty years, continuous improvements in this technique have allowed a reduction in the temporal resolution regime from milliseconds (Genick et al., 1997) to picoseconds (Schotte et al., 2012). In addition, Laue crystallography has been predominantly limited to photo-activated reactions, where, unlike diffusionbased reactions, initiation is homogeneous and rapid. However, even in photo-induced reactions the size of the crystals matter since molecules absorb the light as it diffuses through the crystal, causing a decrease in transmission with increasing path length. Thus, smaller crystals provide an advantage, allowing more synchronized reaction initiation throughout the crystal and requiring decreased pump intensities for maximal reaction initiation. Furthermore, the volume of the crystals is so small that in practice all molecules within the crystal can be illuminated by the pump. Owing to the inherent properties of XFELs, SFX has become an ideal technique to perform time-resolved experiments (TR-SFX). Initially, TR-SFX experiments were carried out using the GDVN liquid injector to deliver the crystals into the beam. More recently, Nogly and coworkers have demonstrated for the first time that time-resolved pump-probe serial crystallography is also feasible using a high-viscosity injector (Nogly et al., 2015), allowing a much lower sample consumption. Therefore, the use of a high-viscosity injector in combination with a pink beam will also provide an unprecedented view into the relations between protein structure, dynamics and function at synchrotron sources.

It is important to note that radiation damage is currently inevitable when using synchrotron sources. For decades, cryocooling has been used to mitigate the effect of radiation damage, which is the primary factor limiting the quality of the structural information that can be obtained from a protein crystal (Garman, 2010). Several radiation-damage studies carried out on macromolecular crystals have revealed that the lifetime of the crystals can be prolonged with an increase in the beam intensity and a decrease in exposure time (Owen et al., 2012; Warkentin et al., 2011, 2013). These studies have also shown that global radiation damage occurs on a timescale of seconds even with a $100 \mathrm{~ms}$ exposure. However, radiation damage can be notably reduced by outrunning the secondary and tertiary damage effects (Owen et al., 2012; Warkentin et al., 2011). The new technique of serial crystallography, in which each diffraction pattern is from a different crystal, reduces radiation damage even when measuring crystals at room temperature at synchrotron sources (Coquelle et al., 2015; Nogly et al., 2015; Stellato et al., 2014). No radiation damage was reported in the structures of lysozyme or bacteriorhodopsin from data sets collected using a liquid injector (Stellato et al., 2014) or an LCP injector (Nogly et al., 2015), respectively. The maximum radiation doses were estimated to be 0.3 MGy (Stellato et al., 2014) and 0.7 MGy (Nogly et al., 2015). However, evidence of specific radiation damage was observed at the disulfide bridges of the structure of lysozyme from data sets collected using a raster-scanning method (Coquelle et al., 2015). Considerably higher doses (3.2 and 29.1 MGy) than the theoretical safe dose limit of $0.3 \mathrm{MGy}$ (Nave \& Garman, 2005) were used. A comparison between the lysozyme structures when delivering crystals using a jet (Stellato et al., 2014) and using a fixed target (Coquelle et al., 2015), which were collected on the same beamline (ID-13 at the ESRF) under similar conditions, suggests that the radiation doses per crystal are higher when immobilizing crystals in a solid support and therefore they are more subject to radiation damage. In the study presented here, the LCP injector was operated at an average flow rate of $100 \mathrm{nl} \mathrm{min}^{-1}$, which provided a constant stream of fresh crystals, so that the average exposure time per crystal was estimated to be about $30 \mathrm{~ms}$. The average total radiation dose that each single crystal received was estimated to be only $0.1 \mathrm{MGy}$, which is lower than the safe dose limit reported by Garman and coworkers for room-temperature measurements (Garman \& McSweeney, 2007; Nave \& Garman, 2005). In fact, we could not detect any sign of radiation damage on investigating the radiationsensitive residues in the structures of either $\mathrm{A}_{2 \mathrm{~A}} \mathrm{AR}$ or lysozyme, demonstrating that the methods described here enable data collection at room temperature with minimal radiation damage.

SMX experiments can also be performed at cryogenic temperatures to reduce radiation damage by raster scanning and oscillation of microcrystals mounted into fixed targets (Gati et al., 2014; Hasegawa et al., 2016; Zander et al., 2015), allowing crystals to be exposed for longer. Also, since microcrystals are rotated during data collection, SMX at cryogenic temperatures allows complete data to be collected using a much lower sample consumption compared with that of the room-temperature-based method. However, SMX at room temperature offers several advantages over the cryo-based method, namely (i) room-temperature data collection eliminates the need for cryoprotection, which is often difficult and in some in cases impossible because the cryocooling process itself can adversely reduce the crystalline order or affect the protein structure (Fraser et al., 2009, 2011; Keedy et al., 2014), and (ii) as mentioned above, SMX at room temperature opens the door to time-resolved pump-probe experiments in the 
near future. By contrast, SMX using cryocooled crystals is only limited to the determination of static structures.

Finally, our study also confirms that for strongly diffracting crystals such as lysozyme, the serial crystallography approach at current synchrotron sources provides crystal structures of reasonable quality for crystals of $5 \mu \mathrm{m}$ and larger. However, for weakly diffracting crystals with larger unit cells such as $\mathrm{A}_{2 \mathrm{~A}} \mathrm{AR}$ or $\mathrm{PC}$, the serial approach at room temperature does not yield a resolution as high as can be obtained at XFELs or from cryocooled crystals. Weakly diffracting crystals or crystals with large unit cells will strongly benefit from further upgrades of synchrotron sources and increased X-ray flux.

\section{Acknowledgements}

This work was supported by the Centre for Applied Structural Discovery (CASD) at the Biodesign Institute at Arizona State University (WL, PF, UW, JCHS, JMMG, CEC, GN, NS, NAZ, LZ, NN, SRC, JZ, GS, JC, AS and DJ), a Mayo Clinic ASU Collaborative Seed Grant Award (WL), the Flinn Foundation Seed Grant (WL) and No. 1991 (PF, JCHS and JMMG), the STC Program of the National Science Foundation through BioXFEL (No. 1231306; WL, PF, UW, JCHS, CEC, GN, NS, NAZ, SRC, GS, JC, AS, DJ and GK), National Science Foundation BIO ABI grant No. 1565180 (NAZ, UW and JCHS), the National Institutes of Health PSI:Biology Center for Membrane Proteins in Infectious Diseases (U54 GM094599; PF, JMMG, NN and JZ), National Institutes of Health grant R21DA042298 (WL and LZ), the National Institute of Health Femtosecond Nano-crystallography of Membrane Proteins (No. R01GM095583; PF and CEC) and National Institutes of Health grants R01GM108635 (VC and AI) and U54GM094618 (VC and AI). Use of the GM/CA at the Advanced Photon Source (APS), Argonne National Laboratory was supported by the National Cancer Institute (ACB-12002; RFF, DK, MCH, CO, NV, SX, SC and DF) and the National Institute of General Medical Sciences (AGM12006; RFF, DK, MCH, CO, NV, SX, SC and DF). Use of the Advanced Photon Source, a US Department of Energy (DOE) Office of Science User Facility operated for the DOE Office of Science by Argonne National Laboratory, was supported under Contract No. DE-AC02-06CH11357.

\section{Funding information}

Funding for this research was provided by: Flinn Foundation (award No. 1991); The STC Program of the National Science Foundation through BioXFEL (award No. 1231306); National Science Foundation BIO ABI grant (award No. 1565180); National Institutes of Health (award Nos. U54GM094599, R01GM095583, R01GM108635, U54GM094618); National Cancer Institute (award No. ACB-12002); National Institute of General Medical Sciences (award No. AGM-12006).

\section{References}

Adams, P. D. et al. (2010). Acta Cryst. D66, 213-221.

Ayvazyan, V. N. et al. (2006). Eur. Phys. J. D, 37, 297-303.
Bailey, F. E. Jr (1976). Poly(ethylene Oxide). New York: Academic Press.

Barty, A., Kirian, R. A., Maia, F. R. N. C., Hantke, M., Yoon, C. H., White, T. A. \& Chapman, H. (2014). J. Appl. Cryst. 47, 1118-1131. Batyuk, A. et al. (2016). Sci. Adv. 2, e1600292.

Bianconi, P. A., Lin, J. \& Strzelecki, A. R. (1991). Nature (London), 349, 315-317.

Blank, Z. R. A. C. \& Reimschuessel, A. C. (1974). J. Mater. Sci. 9, 1815-1822.

Botha, S., Nass, K., Barends, T. R. M., Kabsch, W., Latz, B., Dworkowski, F., Foucar, L., Panepucci, E., Wang, M., Shoeman, R. L., Schlichting, I. \& Doak, R. B. (2015). Acta Cryst. D71, 387-397.

Boutet, S. et al. (2012). Science, 337, 362-364.

Caffrey, M. \& Cherezov, V. (2009). Nat. Protoc. 4, 706-731.

Chandrasekhar, R. (2000). J. Mater. Sci. Lett. 19, 1801-1803.

Chapman, H. N. et al. (2011). Nature (London), 470, 73-77.

Cheng, A., Hummel, B., Qiu, H. \& Caffrey, M. (1998). Chem. Phys. Lipids, 95, 11-21.

Choquesillo-Lazarte, D. \& García-Ruiz, J. M. (2011). J. Appl. Cryst. 44, 172-176.

Conrad, C. E. et al. (2015). IUCrJ, 2, 421-430.

Coquelle, N., Brewster, A. S., Kapp, U., Shilova, A., Weinhausen, B., Burghammer, M. \& Colletier, J.-P. (2015). Acta Cryst. D71, 11841196.

Csizmók, V., Bokor, M., Bánki, P., Klement, É., Medzihradszky, K. F., Friedrich, P., Tompa, P. \& Tompa, P. (2005). Biochemistry, 44, 39553964.

Denning, D. P., Patel, S. S., Uversky, V., Fink, A. L. \& Rexach, M. (2003). Proc. Natl Acad. Sci. USA, 100, 2450-2455.

Dhawan, S., Dhawan, K., Varma, M. \& Sinha, V. R. (2005). Pharm. Technol. 29, 82-95.

Duisenberg, A. J. M. (1992). J. Appl. Cryst. 25, 92-96.

Emsley, P. \& Cowtan, K. (2004). Acta Cryst. D60, 2126-2132.

Feld, G. K. et al. (2015). J. Appl. Cryst. 48, 1072-1079.

Fischetti, R. F., Xu, S., Yoder, D. W., Becker, M., Nagarajan, V., Sanishvili, R., Hilgart, M. C., Stepanov, S., Makarov, O. \& Smith, J. L. (2009). J. Synchrotron Rad. 16, 217-225.

Fischetti, R. F., Yoder, D., Xu, S., Makarov, O., Ogata, C. \& Smith, J. L. (2013). J. Phys. Conf. Ser. 425, 012006.

Fraser, J. S., Clarkson, M. W., Degnan, S. C., Erion, R., Kern, D. \& Alber, T. (2009). Nature (London), 462, 669-673.

Fraser, J. S., van den Bedem, H., Samelson, A. J., Lang, P. T., Holton, J. M., Echols, N. \& Alber, T. (2011). Proc. Natl Acad. Sci. USA, 108, 16247-16252.

Fromme, R., Ishchenko, A., Metz, M., Chowdhury, S. R., Basu, S., Boutet, S., Fromme, P., White, T. A., Barty, A., Spence, J. C. H., Weierstall, U., Liu, W. \& Cherezov, V. (2015). IUCrJ, 2, 545551.

Garman, E. F. (2010). Acta Cryst. D66, 339-351.

Garman, E. F. \& McSweeney, S. M. (2007). J. Synchrotron Rad. 14, $1-3$.

Gati, C., Bourenkov, G., Klinge, M., Rehders, D., Stellato, F., Oberthür, D., Yefanov, O., Sommer, B. P., Mogk, S., Duszenko, M., Betzel, C., Schneider, T. R., Chapman, H. N. \& Redecke, L. (2014). IUCrJ, 1, 87-94.

Genick, U. K., Borgstahl, G. E. O., Ng, K., Ren, Z., Pradervand, C., Burke, P. M., Srajer, V., Teng, T.-Y., Schildkamp, W., McRee, D. E., Moffat, K. \& Getzoff, E. D. (1997). Science, 275, 1471-1475.

Hajdu, J. \& Johnson, L. N. (1990). Biochemistry, 29, 1669-1678.

Hammouda, B., Ho, D. L. \& Kline, S. (2004). Macromolecules, 37, 6932-6937.

Hasegawa, K., Yamashita, K., Murai, T., Nuemket, N., Hirata, K., Ueno, G., Ago, H., Nakatsu, T., Kumasaka, T. \& Yamamoto, M. (2017). J. Synchrotron Rad. 24, 29-41.

Hau-Riege, S. P. \& Bennion, B. J. (2015). Phys. Rev. E, 91, 022705.

Heymann, M., Opthalage, A., Wierman, J. L., Akella, S., Szebenyi, D. M. E., Gruner, S. M. \& Fraden, S. (2014). IUCrJ, 1, 349-360. 
Hilgart, M. C., Sanishvili, R., Ogata, C. M., Becker, M., Venugopalan, N., Stepanov, S., Makarov, O., Smith, J. L. \& Fischetti, R. F. (2011). J. Synchrotron Rad. 18, 717-722.

Huang, C.-Y., Olieric, V., Ma, P., Howe, N., Vogeley, L., Liu, X., Warshamanage, R., Weinert, T., Panepucci, E., Kobilka, B., Diederichs, K., Wang, M. \& Caffrey, M. (2016). Acta Cryst. D72, 93-112.

Huang, C.-Y., Olieric, V., Ma, P., Panepucci, E., Diederichs, K., Wang, M. \& Caffrey, M. (2015). Acta Cryst. D71, 1238-1256.

Kabsch, W. (2010). Acta Cryst. D66, 125-132.

Katritch, V., Fenalti, G., Abola, E. E., Roth, B. L., Cherezov, V. \& Stevens, R. C. (2014). Trends Biochem. Sci. 39, 233-244.

Keedy, D. A., van den Bedem, H., Sivak, D. A., Petsko, G. A., Ringe, D., Wilson, M. A. \& Fraser, J. S. (2014). Structure, 22, 899-910.

Kirian, R. A., Wang, X., Weierstall, U., Schmidt, K. E., Spence, J. C. H., Hunter, M., Fromme, P., White, T., Chapman, H. N. \& Holton, J. (2010). Opt. Express, 18, 5713-5723.

Kirian, R. A., White, T. A., Holton, J. M., Chapman, H. N., Fromme, P., Barty, A., Lomb, L., Aquila, A., Maia, F. R. N. C., Martin, A. V., Fromme, R., Wang, X., Hunter, M. S., Schmidt, K. E. \& Spence, J. C. H. (2011). Acta Cryst. A67, 131-140.

Kissick, D. J., Wanapun, D. \& Simpson, G. J. (2011). Annu. Rev. Anal. Chem. 4, 419-437.

Kupitz, C., Grotjohann, I., Conrad, C. E., Roy-Chowdhury, S., Fromme, R. \& Fromme, P. (2014). Philos. Trans. R. Soc. Lond B Biol. Sci. 369, 20130316.

Liu, W., Chun, E., Thompson, A. A., Chubukov, P., Xu, F., Katritch, V., Han, G. W., Roth, C. B., Heitman, L. H., IJzerman, A. P., Cherezov, V. \& Stevens, R. C. (2012). Science, 337, 232-236.

Liu, W., Ishchenko, A. \& Cherezov, V. (2014). Nat. Protoc. 9, 2123 2134.

Lomb, L. et al. (2011). Phys. Rev. B, 84, 214111.

Low, B. W., Chen, C. C. H., Berger, J. E., Singman, L. \& Pletcher, J. F. (1966). Proc. Natl Acad. Sci. USA, 56, 1746-1750.

Macchi, P. (2012). Top. Curr. Chem. 315, 33-67.

Marín, M., Thallmair, V. \& Ott, T. (2012). J. Biol. Chem. 287, 3998239991.

Martin-Garcia, J. M., Conrad, C. E., Coe, J., Roy-Chowdhury, S. \& Fromme, P. (2016). Arch. Biochem. Biophys. 602, 32-47.

Martin-Garcia, J. M., Hansen, D. T., Zook, J., Loskutov, A. V., Robida, M. D., Craciunescu, F. M., Sykes, K. F., Wachter, R. M., Fromme, P. \& Allen, J. P. (2014). Biochemistry, 53, 1958-1970.

McCoy, A. J., Grosse-Kunstleve, R. W., Adams, P. D., Winn, M. D., Storoni, L. C. \& Read, R. J. (2007). J. Appl. Cryst. 40, 658-674.

Murray, T. D., Lyubimov, A. Y., Ogata, C. M., Vo, H., Uervirojnangkoorn, M., Brunger, A. T. \& Berger, J. M. (2015). Acta Cryst. D71, 1987-1997.

Murshudov, G. N., Skubák, P., Lebedev, A. A., Pannu, N. S., Steiner, R. A., Nicholls, R. A., Winn, M. D., Long, F. \& Vagin, A. A. (2011). Acta Cryst. D67, 355-367.

Nave, C. \& Garman, E. F. (2005). J. Synchrotron Rad. 12, 257-260.

Neutze, R., Wouts, R., van der Spoel, D., Weckert, E. \& Hajdu, J. (2000). Nature (London), 406, 752-757.

Nogly, P. et al. (2015). IUCrJ, 2, 168-176.

Nyarko, A., Hare, M., Hays, T. S. \& Barbar, E. (2004). Biochemistry, 43, 15595-15603.

Owen, R. L., Axford, D., Nettleship, J. E., Owens, R. J., Robinson, J. I., Morgan, A. W., Doré, A. S., Lebon, G., Tate, C. G., Fry, E. E., Ren, J., Stuart, D. I. \& Evans, G. (2012). Acta Cryst. D68, 810-818. Pflugrath, J. W. (2004). Methods, 34, 415-423.

Pietras, Z., Lin, H.-T., Surade, S., Luisi, B., Slattery, O., Pos, K. M. \& Moreno, A. (2010). J. Appl. Cryst. 43, 58-63.

Pothineni, S. B., Venugopalan, N., Ogata, C. M., Hilgart, M. C., Stepanov, S., Sanishvili, R., Becker, M., Winter, G., Sauter, N. K.,
Smith, J. L. \& Fischetti, R. F. (2014). J. Appl. Cryst. 47, 1992-1999.

Powell, H. R., Johnson, O. \& Leslie, A. G. W. (2013). Acta Cryst. D69, 1195-1203.

Ravelli, R. B. G. \& Garman, E. F. (2006). Curr. Opin. Struct. Biol. 16, 624-629.

Redecke, L. et al. (2013). Science, 339, 227-230.

Sanishvili, R., Nagarajan, V., Yoder, D., Becker, M., Xu, S., Corcoran, S., Akey, D. L., Smith, J. L. \& Fischetti, R. F. (2008). Acta Cryst. D64, 425-435.

Sauter, N. K. (2015). J. Synchrotron Rad. 22, 239-248.

Sawaya, M. R. et al. (2014). Proc. Natl Acad. Sci. USA, 111, 12769 12774.

Schirmer, T., Bode, W., Huber, R., Sidler, W. \& Zuber, H. (1985). J. Mol. Biol. 184, 257-277.

Schotte, F., Cho, H. S., Kaila, V. R., Kamikubo, H., Dashdorj, N., Henry, E. R., Graber, T. J., Henning, R., Wulff, M., Hummer, G., Kataoka, M. \& Anfinrud, P. A. (2012). Proc. Natl Acad. Sci. USA, 109, 19256-19261.

Sierra, R. G. et al. (2012). Acta Cryst. D68, 1584-1587.

Stellato, F. et al. (2014). IUCrJ, 1, 204-212.

Stepanov, S., Makarov, O., Hilgart, M., Pothineni, S. B., Urakhchin, A., Devarapalli, S., Yoder, D., Becker, M., Ogata, C., Sanishvili, R., Venugopalan, N., Smith, J. L. \& Fischetti, R. F. (2011). Acta Cryst. D67, 176-188.

Su, J., Yang, J., Zhao, D., Kawula, T. H., Banas, J. A. \& Zhang, J. R. (2007). Infect. Immun. 75, 3089-3101.

Terwilliger, T. C., Dimaio, F., Read, R. J., Baker, D., Bunkóczi, G., Adams, P. D., Grosse-Kunstleve, R. W., Afonine, P. V. \& Echols, N. (2012). J. Struct. Funct. Genomics, 13, 81-90.

Terwilliger, T. C., Grosse-Kunstleve, R. W., Afonine, P. V., Moriarty, N. W., Zwart, P. H., Hung, L.-W., Read, R. J. \& Adams, P. D. (2008). Acta Cryst. D64, 61-69.

Vagin, A. \& Teplyakov, A. (2010). Acta Cryst. D66, 22-25.

Warkentin, M., Badeau, R., Hopkins, J. \& Thorne, R. E. (2011). Acta Cryst. D67, 792-803.

Warkentin, M., Hopkins, J. B., Badeau, R., Mulichak, A. M., Keefe, L. J. \& Thorne, R. E. (2013). J. Synchrotron Rad. 20, 7-13.

Weierstall, U. et al. (2014). Nat. Commun. 5, 3309.

Weierstall, U., Spence, J. C. H. \& Doak, R. B. (2012). Rev. Sci. Instrum. 83, 035108.

White, T. A., Barty, A., Stellato, F., Holton, J. M., Kirian, R. A., Zatsepin, N. A. \& Chapman, H. N. (2013). Acta Cryst. D69, 12311240.

White, T. A., Kirian, R. A., Martin, A. V., Aquila, A., Nass, K., Barty, A. \& Chapman, H. N. (2012). J. Appl. Cryst. 45, 335-341.

White, T. A., Mariani, V., Brehm, W., Yefanov, O., Barty, A., Beyerlein, K. R., Chervinskii, F., Galli, L., Gati, C., Nakane, T., Tolstikova, A., Yamashita, K., Yoon, C. H., Diederichs, K. \& Chapman, H. N. (2016). J. Appl. Cryst. 49, 680-689.

Xiang, J., Chun, E., Liu, C., Jing, L., Al-Sahouri, Z., Zhu, L. \& Liu, W. (2016). Trends Pharmacol. Sci. 37, 1055-1069.

Yazawa, K., Sugahara, M., Yutani, K., Takehira, M. \& Numata, K. (2016). ACS Catal. 6, 3036-3046.

Zander, U., Bourenkov, G., Popov, A. N., de Sanctis, D., Svensson, O., McCarthy, A. A., Round, E., Gordeliy, V., Mueller-Dieckmann, C. \& Leonard, G. A. (2015). Acta Cryst. D71, 2328-2343.

Zeldin, O. B., Brockhauser, S., Bremridge, J., Holton, J. M. \& Garman, E. F. (2013). Proc. Natl Acad. Sci. USA, 110, 20551-20556.

Zeldin, O. B., Gerstel, M. \& Garman, E. F. (2013). J. Synchrotron Rad. 20, 49-57.

Zook, J., Mo, G., Sisco, N. J., Craciunescu, F. M., Hansen, D. T., Baravati, B., Cherry, B. R., Sykes, K., Wachter, R., Van Horn, W. D. \& Fromme, P. (2015). Structure, 23, 1116-1122. 